\title{
Energy Efficient for Data Aggregation in Wireless Sensor Networks
}

\author{
Abdulmalik Danmallam Bello \\ Dept. of Electronics and Communication Engineering, \\ Gyan Vihar School of Engneering Technology, \\ Suresh Gyan Vihar University Jaipur, \\ Rajasthan, India.
}

\author{
Dr. O.S. Lamba \\ Dept. of Electronics and Communication Engineering, \\ Gyan Vihar School of Engneering Technology, \\ Suresh Gyan Vihar University Jaipur, \\ Rajasthan, India.
}

\begin{abstract}
The wireless sensor networks is the decentralized and self configuring type of network in which senor nodes can sense information and pass it to base station. Due to decentralized nature and far deployment energy consumption is the major issues of wireless sensor networks. To reduce energy consumption of wireless sensor hierarchal clustering is the efficient type of clustering technique. In this scheme whole network will be divided into fixed size clusters and cluster heads are selected in each cluster. The cluster heads are selected on the basis of energy and distance from base station. The sensor node which has least distance from the base station and has maximum energy is selected as cluster head. The cluster heads can communicate with each other and data will be transmitted to base station. In this research work, WEMER protocol is implemented and improved to increase lifetime of wireless sensor networks. In the WEMER protocol, whole network is divided into clusters and cluster heads are selected in each cluster. The leader nodes are also selected in the network which take data from the cluster heads and pass it to base station. In the improvement of WEMER protocol. Gateway nodes are deployed in network to increase lifetime of WSN. In the proposed improvement gateway nodes are deployed near to base station which takes data from the leader nodes. The leader nodes take data from the cluster head. The proposed WEMER protocol and WEMER protocol are implemented in MATLAB. The simulation results shows that proposed WEMER protocol has less number of dead nodes, high number of alive nodes, send more number of packets and more remaining energy consumption.
\end{abstract}

\section{CHAPTER 1 \\ INTRODUCTION}

\subsection{Introduction to Wireless Sensor Networks}

A wireless sensor network is defined as the network in which there are large numbers of sensor nodes deployed such that important information from the surrounding regions can be collected and processed. The size of sensor node present in the network is very small. Thus, the processing capabilities it provides and the battery available in it is very constraint. According to the changes, the information is collected from the surroundings and then forwarded further by the network. This network has huge computational and processing constraints. Motes are referred to as the small sized computers with the help of which information is collected from all across the region. Motes are deployed today within various industrial applications. Using a group of motes, certain objectives within an application are accomplished by collecting all the information related to the activities being performed in the surroundings [1]. Depending on variety of configurations, motes are connected with each other through inks such that the best performance results can be achieved. In order to perform communication amongst each other, transceivers are used by motes. The deployment of sensor network within an application can include around hundreds to thousands of sensor nodes. However, there are comparatively less numbers of sensor nodes present within the ad hoc networks which do not include any infrastructure.

For generating a sensing unit within a sensor node, several sensors are combined together. Any kind of important information collected from the surroundings by the sensing unit present in sensor nodes, is passed on to the processing unit such that it can be processed and be efficiently used. Any kinds of analog signals created from sensors are digitized using Analog to Digital converter (ADC). The signal is forwarded to the controller such the processing can be performed. Another important part of a sensor node is its processing unit since the execution and functioning of several tasks is done with the help of processor present within it. The pre-programming and loading of processing unit is done through the services that are provided by the sensor nodes. The rate at which energy is to be consumed is different for the processor depending upon the functionality of nodes. The difference if the performance of a processor can easily be identified using several evaluating factors like memory, data rate and processing speed. The computations in a processing unit can be performed easily by the communication unit. The outcomes achieved are forwarded to the base station such that they can be sent to external users. It is possible to transmit or receive the information using a basic transceiver that acts as a communication unit in between the nodes and base station. The four important stages of communication unit are transmit, receive, idle and sleep. 


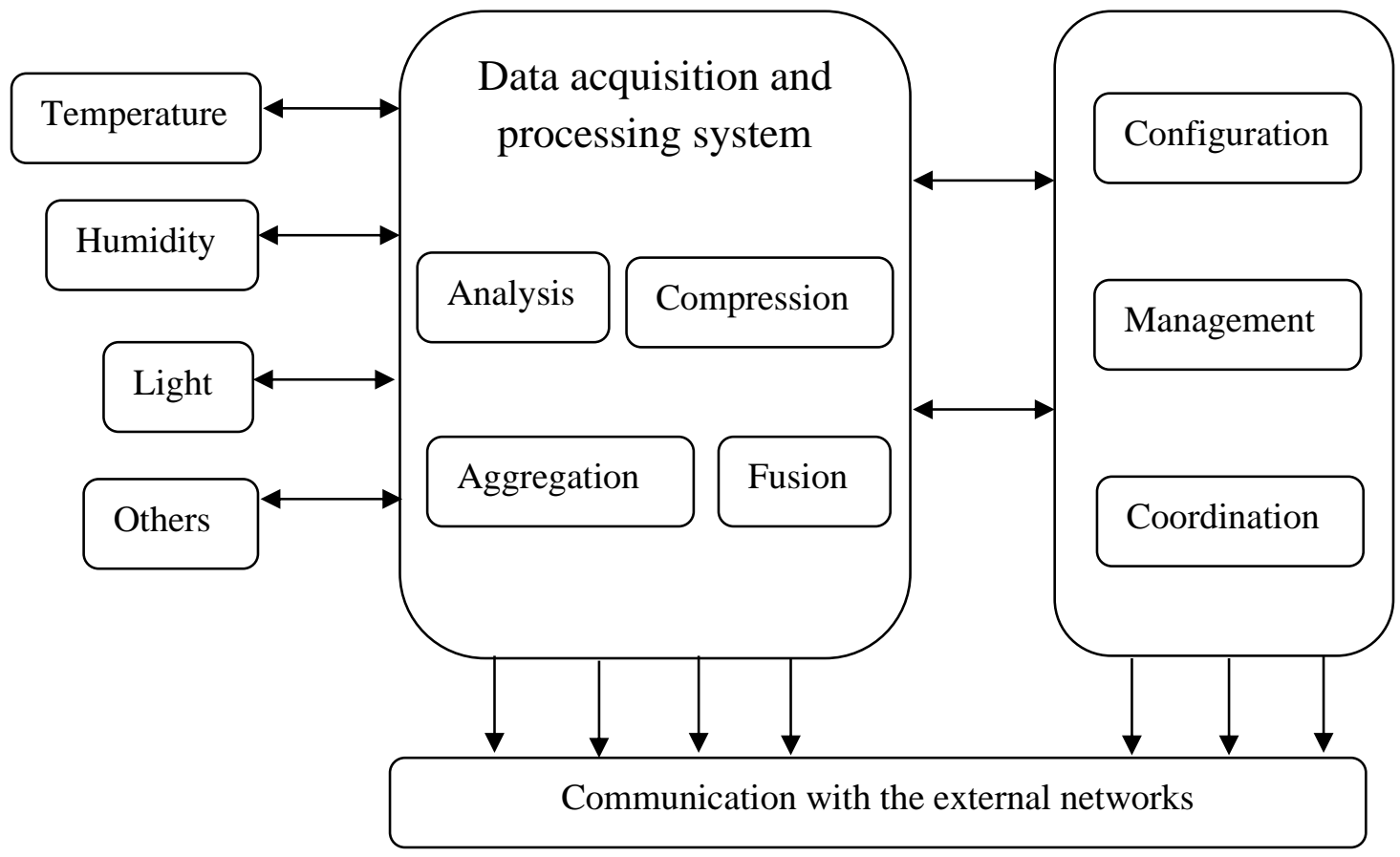

Figure 1.1 Functionality of a Sensor Node

Figure 1.1 functionality of a sensor node

Following are some of the important properties of sensor nodes that help in evaluating the performance of WSN:

i. Fault tolerance: Within each node present in the network, an unanticipated failure is possible to occur [2]. Fault tolerance is defined as the approach through which the common functionalities of sensor networks are ensured to be continuing even though a sensor node failure occurs.

ii. Mobility of nodes: On the basis of applications, the sensor nodes are free to move within the area of deployment. This results in increasing the efficiency of communication in networks.

iii. Dynamic network topology: On the basis of connection that is established amongst the sensor nodes, a standard topology is set up in the network applications. The performance of WSNs can also be enhanced using dynamic topology within them.

iv. Communication failures: The information related to any kinds of node failure within the network is important to be informed to the base station.

v. Heterogeneity of nodes: Since there are several sensor nodes deployed within the WSN applications, it is important to ensure that they work in a cooperative fashion with each other to perform certain tasks.

vi. Scalability: In a network, there are hundreds to thousands of sensor nodes available. Higher scalability of these networks is important to be ensured.

vii. Independency: In order to perform different tasks in these networks, no central authority is required here.

viii. Programmability: These networks require reprogramming or reconfiguration in order to increase the adaptability to dynamic changes occurring in them.

ix. Utilization of sensors: It is important to ensure that minimum energy is consumed by sensor nodes such that highest performance results are achieved.

x. Impracticality of public key cryptosystems: Since the computation and power resources are very limited, it is not possible to use public key algorithms in these networks.

xi. Lack of a prior knowledge of post-deployment configuration: When there is a random distribution of sensor nodes in the network, it is not possible to known the communication status of nodes.

\subsubsection{Network Characteristics}

There are several sensor nodes deployed in the region of interest when a WSN is deployed. The sensor nodes are less costly, small in size and also perform multifunctioning. Even when the size of sensor nodes is small, the processing capabilities are performed [3]. Short distance communications are provided by the wireless medium and nodes are gathered together such that a common task can be accomplished. WSNs have few unique characteristics such that they are different from other networks. Below are few of such characteristics:

- Dense Node Deployment: The sensor nodes are deployed in common manner within the region of interest. There are higher numbers of sensor nodes deployed within these networks as compared to mobile ad hoc networks.

- Battery Powered Sensor Nodes: The sensor nodes are provided with power with the help of batteries deployed within them. Most of the WSN networks 
are deployed within hostile areas and in those areas it is impossible to either recharge or replace the batteries once the complete power is consumed.

- Severe Energy, Computation, and Storage Constraints: The sensor nodes have very limited amount of energy amongst which the computation and storage capabilities consume highest amount of energy.

- Self-Configurable: The deployment of sensor nodes does not require any planning or engineering. Within the areas, the nodes are deployed randomly. The sensor nodes rearrange themselves depending upon the application as they have the property of self-configuration [4].

- Application Specific: On the basis of application, the networks are designed and deployed. The design requirements of the network also change completely depending upon the application in which they are involved.

- Unreliable Sensor Nodes: The sensor nodes are deployed in various hostile areas and to perform certain operations, it is not important to require their attendance. These networks can possibly face various physical damages and failures.

- Frequent Topology Change: Due to the channel fading or node failure, the topology of network changes more frequently.

- No Global Identification: Since there are large numbers of sensor nodes available, creating a global address mechanism for sensor network is impossible. In these sensor networks, the overhead and identity maintenance is higher here.

\subsubsection{Issues and Challenges in Designing WSN}

During the design of WSN, various challenges are being faced. Following are some of these challenges:

i. Since the scattering of nodes is done during their deployment, no permanent topology definition is required here.

ii. Few resources are provided in these networks which result in:

- Limitation of available memory.

- Limitation of calculations.

- Unreliability of the communication being performed.

- Limitation of power available.

iii. There is no infrastructure available within these networks due to which their maintenance is difficult which results in:

- Unreliability of data transmission.

- Occurrence of conflicts and latency.

iv. The power provided in these networks is provided from batteries deployed in them which are impossible to be replaced or recharged due to their deployment in such regions in which human reach is not possible.

v. The hardware design of sensor nodes is important to be considered because [5]:
- Several unattended operations are provided by them.

- It is possible that physical attacks occur.

- There is remote management of these networks.

- In these networks, no central authority is available.

vi. It is important for the sensor nodes to be synchronized in these networks which are also another issue to be considered.

vii. It is possible for the nodes to be added or removed or node failure might occur as a result of which the network topologies might change.

viii. Because these networks have different transmission nature and hostile environment, security is an important challenge being faced.

ix. On the basis of types of applications and types of computations involved, the selection of sensor nodes is possible.

\subsubsection{Network Applications}

The sensors deployed within these networks make it possible to monitor or recognize the physical conditions in WSNs. These networks have more benefits in comparison to the conventional wired networks. It is not possible to reduce all the costs and delays occurring within these networks. The deployment of WSNs is done within several regions ranging from military applications to other small range of applications as well. Since the sensor nodes involved within these networks are very less costly and they provide ease of communication, WSNs can be easily deployed within several applications such as in military and civil regions. Following are some of the applications in which these networks have been deployed:

\section{a. Environmental Monitoring}

The surveillance of environments is one of the most prior objectives of WSNs and the very first application in which they were deployed was in military applications [6]. The surroundings include several changes in the environmental conditions which need to be monitored by these sensors.

- Habitat Monitoring: The sensors help in monitoring the conditions of animals or the plants and also gather information of other habitat present in those regions.

- Air or Water Quality Monitoring: The deployment of sensors is done in order to monitor the quality of water and air within particular areas. For instance, the quality of water is kept under surveillance in various hydrochemistry regions. With the help of these networks, the quality of air can be monitored such the air pollution can be controlled properly.

- Hazard Monitoring: The deployment of sensors also helps in monitoring any kinds of emergency situations arising within biological or chemical applications.

- Disaster Monitoring: These networks are also deployed within regions such that any natural or manmade disasters can be monitored and informed to concerned authorities. For instance, 
the sensors are distributed all across the forest areas such that any kinds of forest fires or floods can be recognized. Any quake's direction or magnitude can be recognized such that the people in buildings can be saved.

\section{b. Military Applications.}

There are several important roles to be played by WSNs within several applications such as in military or telecommunication systems. Since they are easy to be deployed are self-controllable and can also provide fault tolerance in emergency conditions, the C3I systems also deploy such kinds of networks. There is reduction of humans within the wars due to the deployment of such networks and several intelligent systems have been advanced through these networks.

- Battlefield Monitoring: The battlefields are deployed with various sensor nodes such that the forces and military vehicles present in those regions can be monitored at all times. There is a close monitoring of the mobility of all such vehicles such that any kinds of information related to their actions can be gathered.

- $\quad$ Object Protection: These sensor nodes also help in protecting the regions such that any kinds of sensitive objects can be monitored at all time durations within certain applications.

- Intelligent Guiding: There is a need to maintain coordination amongst the robotic vehicles, military tanks, and missiles such that a defensive attack can be performed against opposition party; the deployment of these networks is used.

- Remote Sensing: Through the identification of any chemical weapons or nuclear weapons, by deploying sensors in regions it is possible to prevent terrorist attacks to occur.

\section{c. Health Care Applications.}

Various health departments have lack of health care personnel due to which the health of patients are at risk. Thus, the sensor networks are deployed within such hospitals so that the patients can be monitored at all times and the help required can be provided to them [7]. Thus, there is reduction in cost expenditures of these health care systems as well.

- Behavior Monitoring: The deployment of sensors is done within homes such that the behaviors of patients or normal people can be done to keep a check on their activities. The doctors are informed regarding any kind of emergency situation arising here. The sensors pass the instructions or reminders to the patients by keeping a track on their activities.

- Medical Monitoring: Wireless body area networks (WBANs) are deployed within the homes of old age people to keep a track on their daily activities. Within several other applications as well such as the hospital buildings, these networks are deployed so that a proper monitoring of all the patients can be done and their medication can be given according to the requirement. The doctors are informed regarding any kind of emergency conditions and immediate actions are taken as per the need. Further, to keep a track regarding the health improvement of a patient, its tracks over certain period of time are maintained which can also be done here.

\section{d. Industrial Process Control.}

With the help of deploying WSNs, it is possible to manufacture the devices or the conditions in which the industries are needed to be generated. For instance, the wireless sensors can help in monitoring and controlling the production process in the assembly and production lines. The chemical regions or oil refiners use the sensors such that the conditions of pipelines can be monitored. It is not possible to monitor the conditions of machinery from the regions that are out of reach of humans. Thus, any kinds of failures in such regions can be identified and preventive measures can be taken accordingly. For preventing further any failures, the sensors are deployed such that the actions can be tracked. A schedule commonly is set up here as per which the machinery is checked with the time interval such that the equipment can be maintained. However, the cost of such systems is higher. The sensor networks maintain the devices on the basis of the conditions of the equipment. It is possible to minimize the cost of maintenance which can further result in increasing the lifetime of machines.

\section{e. Security and Surveillance.}

The deployment of WSNs is done within several security and surveillance applications. For example, the acoustic or video sensors are deployed in the buildings, airports and underwater applications such that the important information can be gathered. In these applications it is possible to identify and track any kinds of intruders and for preventing any failures, various protection techniques are applied within these applications [8]. These applications also do not require any fixed infrastructure. Various security applications are set up in the systems such that power and communication facilities can be provided in these networks.

\section{f. Home Intelligence.}

Through the deployment of WSNs in various applications, a convenient and intelligent living condition is introduced. Some of such applications include:

- Smart Home: By deploying wireless sensors in the homes and linking them with each other, an autonomous network is created at homes. For instance, using the inventory present within the refrigerators, a menu can be created by making a direct connection within the smart refrigerator and the smart stoves.

- Remote Metering: The deployment of WSNs is done such that the utility meters available within the home can be read remotely. For instance, the readings of water, gas or electricity can easily be taken remotely through the sensors and provided to user through wireless communication. 


\subsection{Energy Consumption in WSN}

The WSNs consist of un-attended and un-tethered sensor nodes within them which help in monitoring the surroundings. Within the area of interest, the distribution of these sensor nodes is done and multi-hops are used to transfer such information further [9]. With the help of such deployments, an ad hoc network is created in this manner. The sensor nodes include within them the battery powers which are small in size and irreplaceable or rechargeable. The nodes collect important information which is processed and stored within the sink node or the gateway node which is usually one within the region. An important component of sensor nodes is battery which helps in performing data acquisition. It is however, not possible to recharge these batteries. The batteries include few energy generating units which are known as photo-voltaic cells within them. Due to the node acquisition the energy of order 1 to $2 \mathrm{~J}$ is provided as there are very small sized sensor nodes present in the networks. Thus, there is limited lifetime of a sensor and because of this, the overall performance of network also gets affected.

The energy of nodes of WSN is the most important factor to be considered. For increasing the lifetime of networks, it is important that the energy can be saved from hardware and software solutions provided in the network. As per the various researches made on this network, it is seen that data communication consumes higher amount of energy in comparison to other activities being performed in the network [10]. Thus, there is a need of only short range communications in these networks such that less transmission power is being consumed. The long-range communications are avoided in these networks. The sensing of events by WSNs near to the region of interest and far from the sink nodes is also possible. therefore, for forwarding data packets over larger distances, multi-path communication is required with the help of intermediate nodes.

\subsection{Routing Protocols in WSN}

It is very different to perform routing within WSNs as compared to the conventional routing that is performed in fixed networks. As the network does not include any infrastructure, the wireless links provided are unreliable. The routing protocols provide node failures here due to which it is important to save energy in the networks. Several researchers have introduced various routing protocols which have been categorized into various categories. All such categories have been presented below:

\section{A. Location-based Protocols}

The location-based protocols mainly focus on the information related to the location of sensor nodes. The location of sensor nodes is important to be known by most of the routing protocols such that the distance amongst two particular nodes can be calculated. This mechanism also helps in calculating an estimate of amount of energy that is being consumed by the networks. The researchers have proposed several such protocols amongst which few have been described below:

i. Geographic Adaptive Fidelity (GAF): The Mobile ad hoc networks initially proposed this protocol. Due to the benefit of providing energy conservation, this type of protocol is used by WSNs as well. The GAF protocol is introduced on the basis of energy model through which energy consumption is known [11]. Along with recognition of availability of incoming packets when radio sensor is working, it is possible to transmit and receive the packets in these networks. GAF turns off any kinds of unnecessary sensors available in the network. This also ensures the maintenance of constant level of routing fidelity. The GAF helps in dividing complete sensor field into grid squares and each sensor provides the information that is relevant to the location of the node. The GPS of other location techniques helps in providing location information of the sensor node such that it can be associated with a particular grid. Further, the association is exploited with the help of equivalent sensors with respect to the packet forwarding.

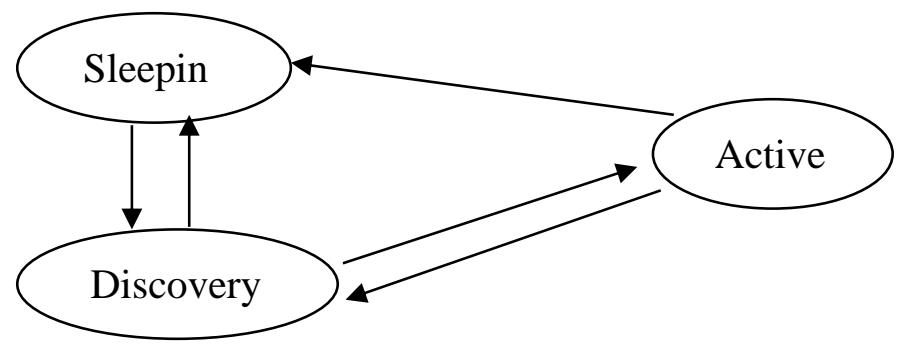

Fig. 1.2: State transition diagram of GAF

The above figure 1.2 shows the three different stages of GAF which are discovery, active and sleeping. When a sensor is in a sleeping stage, the radio is turned off such that the energy can be saved. For identifying the sensors present in similar grid in the discovery stage, the sensors exchange the discovery messages amongst each other. There is a periodic broadcasting of the discovery message even in the active state, when a sensor node is informed related to its state. The amount of time that is consumed by all of the states is tuned by the application. However, it is important to consider few of the important factors such as requirement and sensor mobility. The major objective here is to increase the lifetime of network [12].

ii. Geographic and Energy-Aware Routing (GEAR): This protocol is proposed such that the queries can be routed to the target regions present in the sensor field. The sensors need to equip the localized hardware here. It is important to know the current positions of sensor nodes. The sensors 
must know the locations and residual energy of their neighboring nodes here. GEAR also uses the energy aware heuristics such that the sensors that route the packet forward towards the destination can be selected. The recursive geographic forwarding algorithm is used such that the packet can be disseminated in the target region.

iii. Coordination of Power Saving with Routing: SPAN is another routing protocol which was initially used within MANETs. In WSNs, the amount of energy consumed was reduced through this protocol. This protocol was introduced depending upon the fact that the higher amount of power is consumed by wireless network interface of a device. Another better solution for the idle time was to turn off the radio [13]. During the utilization of geographical forwarding protocol SPAN performs in a much better way although there is no need of location of sensors here. As the packets are forwarded through SPAN instead of the sensors available in between the source and destination, there is a need to join the forwarding backbone topology in the networks. The neighbors and coordinators need to be informed regarding the status of each sensor. Along with SPAN, the election rule is used along with geographic forwarding protocol to collect information related to the status. Any coordinator that is closest to the destination forwards the packet to neighbor once the packet is received by it.

iv. Trajectory-Based Forwarding (TBF): This protocol is used such that a dense network can be deployed and a coordinate system can be generated in the application. The system can be self-localized and it is also possible to estimate the distance of neighbors here. The source mentions the trajectory within the packet. Although, it is not possible to identify the path depending on the hop-byhop communication. With the help of location information about the neighbors, a greedy decision is made here. This helps in knowing the next hop that is closes to the trajectory. The route maintenance amongst TBF is not affected by the mobility of sensors when a trajectory node is involved such that no forwarding nodes are provided.

v. Bounded Voronoi Greedy Forwarding [BVGF]: This is a type of protocol in which similar to the Voronoi diagrams, the geographical positions of sensors are considered to be very important. With the help of sites given in the diagram, the locations of sensors are represented in this protocol. The neighbor that has minimum distance towards the destination is forwarded the packet here. The greedy geographic routing approach is used to do so [14]. For the sensors that can easily act as next hops, the Voronoi areas are traversed by the segment line from which the source and destination are joined. BVGF selects the neighbor that has the shortest Euclidean distance towards the destination from all the neighbors that are eligible to be selected as next hop.

vi. Geographic Random Forwarding (GeRaF): A routing protocol in which a prior to the sensor that acts as relay is not known by the sender is known as GeRaF routing protocol. During all the time intervals, the forwarding of message to the sink is not ensured here. Therefore, besteffort forwarding protocol is another name for this type of protocol. Depending on the assumption of this protocol, the physical locations of all sensors available and sink are known in this routing protocol.

\section{B. Data Centric Protocols}

These types of routing protocols are very different from others as from source to the sink the data is transmitted here. Each source sends the data to the sink in an independent form such that these address-centric protocols can process it. The data that originates from multiple source sensors is aggregated using the data-centric protocols when the transmission is to be performed amongst the source and sink of the network. The amount of energy being saved here is higher as very less amount of transmission from source to sink is needed here. Following are few of these types of protocols:

i. Sensor Protocols for Information via Negotiation (SPIN): In order to improve the classic flooding protocols and solve several issues related to routing, SPIN protocol has been introduced [15]. The two categories of this protocol are resource aware and resource adaptive. With the help of sensors that are available within these protocols, the energy that is required to compute, transmit and receive the data from network is consumed. The decisions are made here therefore, by using the own resources in an efficient way. The two approaches in which these protocols are used are the negotiation and resource adaptation.

ii. Directed Diffusion: The introduction of directed diffusion protocol is done such that sensor query dissemination and processing can be provided to the networks. This protocol helps in fulfilling several requirements of WSN. This approach utilizes data naming, data propagation, and interests etc. as elements within them [16].

\section{Hierarchical Protocols}

Different researchers have proposed several viewpoints on the basis of which hierarchical clustering in WSN has been evaluated and developed. Clustering is the effective communication protocol used to transmit the sensed data towards sink. This study presents a view over the sample of layered protocols involved within various clusters of sensors. Each cluster includes within it a special node known as cluster head. There are few special activities performed by this cluster head for other nodes of the cluster.

The hierarchical approach introduced here generates various clustered layers. When the nodes are grouped to create clusters, a cluster head is chosen amongst them. Towards other base stations or cluster head routing from one cluster is performed. The data is forwarded to higher layer from lower clustered layer [17]. Following are few of these types of routing protocols:

i. Low-energy adaptive clustering hierarchy (LEACH): When the energy efficient clustering algorithm called LEACH is applied within the network, there is reduction in the overall energy being consumed by it. The clustering task is rotated amongst the nodes on the basis of time duration left or energy remaining for a sensor node. Direct communication is provided for each cluster head such that the data can be forwarded to the base station. The clusters 
are used to increase the lifetime of WSNs. Aggregation is known as the mechanism through which the original data is combined from small grid sized data that is forwarded to the individual sensors. The LEACH protocol uses this aggregated data [18].

ii. Power-Efficient Gathering in Sensor Information Systems (PEGASIS): By creating chains of sensor nodes in this manner that the neighbors can transmit and receive the data from neighbors, the LEACH protocol is enhanced. It is named as PEGASIS. From the created chain, only one node is selected such that the data can be transmitted towards the base station. The data is forwarded from one node to the next and then the base station receives this protocol at the end. For constructing the chains such that one sensor dies, similar greedy mechanism is used only with the exclusion of failure sensor. This is done because of the depletion of power of batteries present in the nodes. After each round, the aggregated data is transmitted to the base station through the random selection of sensor node from the chain generated [19]. Therefore, there is less amount of energy consumption as per each round in comparison to LEACH protocol.

iii. Hybrid, Energy-Efficient Distributed Clustering (HEED): Within cluster selection process of HEED, the residual energy and node degree are used as metrics such that a power balance condition can be achieved. This protocol is basically an enhanced version of LEACH. For providing an inter-cluster communication and also providing multi-hop networking operations, the adaptive transmission power is used.

iv. Threshold Sensitive Energy Efficient Sensor Network Protocol (TEEN): TEEN is known as the grouping of sensors into clusters in which individual cluster heads are present. With the help of sensors available in the cluster, the information that is sensed is aggregated to the CHs. The $\mathrm{CH}$ of higher level is then forwarded this information and the process keeps continuing in this manner till the base station receives this data. TEEN is introduced depending upon the hierarchical grouping that creates the clusters using nodes that are near to each other. TEEN is only applied within the applications that have a proper maintenance of the energy efficiency, accuracy and response time.

v. Adaptive Periodic Threshold Sensitive Energy Efficient Sensor Network Protocol (APTEEN): APTEEN is introduced by integrating LEACH and TEEN. This protocol helps in solving issues that arise using TEEN protocol. In this protocol, there is periodic transmission of sensed data and information related to any kinds of modifications within the value of sensed attribute is provided to the CHs. The architectural designs of TEEN and APTEEN are however much alike [20].

\subsection{Review of Literature}

Ramin Yarinezhada, et.al (2018)presented the relationship shared between the sensor nodes and sink node as it forwarded the more traffic loads in the wireless sensor network. This huge traffic leads to depletion of large amount of energy. Therefore, in the network, there is partition between the nodes and the sink isolation. In order to solve the above mentioned issue, they utilized the mobile sink in this network. The appropriate use of the mobile sinks within the network creates the balance in the consumption of energy. Sensor nodes must know the position of the mobile sink in advance which is required to transfer the data to the network [21]. In case, the sensor nodes know the position of the sink in the network, there is more consumption of energy and delay in the network. On the basis of virtual grid infrastructure and mobile sink, they proposed a routing algorithm in this paper. The nodes are selected from the network using, proposed method and with the use of virtual infrastructure, which help in maintaining the position of the sink. They performed experiments and concluded results shows the effectiveness of the proposed method in terms of performance, energy efficient and compared delay as compared to the other methods.

Ram Murthy Garimella, et.al (2018)presented the wireless sensor network in this paper, in which essential role is played by the energy efficiency. This issue is considered as the major in this network due to which various methods has been proposed by the researchers so far. Clustering the widely utilized technique was used in these algorithms but it does not show the mathematically validation. Hence, in order to fill the research gap, they proposed a system and method in this paper. For the process of data gathering and routing in the wireless sensor network, the concept of the Hessian matrix was used for the utilization of efficient energy techniques in this paper [22].This concept was utilized for the validation of the proposed method having the multi variable calculus. On the basis of assumption, in the multidimensional space, these wirelesses sensor nodes are distributed. The performed experiments evaluated the proposed method and illustrated that it can be utilized in any clustering approach using its mathematical design and provide the low-energy communication structure.

Deepa PUNEETH, et.al (2018)presented the study related to some parameters that plays an essential role in the functionality of the wireless sensor network such as energy efficiency, data reliability, and security. The combination of the cryptographic secret sharing technique and the disjoint multipath routing scheme must be used to make a effective and efficient approach that address all thethese requirements. The Shamir's secret sharing (SSS) provided the reliability and information-theoretic security but not able to provide the energy efficiency and optimal results linked to it. In order to obtain the energy efficiency and data reliability, they provided the Shamir's ramp secret sharing (SRSS) in this paper [23]. The compromised node (CN) attack is the limitations of these approaches which occur when there are minimum numbers of nodes are compromised. Therefore, to overcome all the above mentioned issues, they proposed another method in this paper using which security against $\mathrm{CN}$ attacks is provided. It also provided the data reliability, efficient energy in the network. The split hop AES (SHAES) defined the integration of SRSS and a round-reduced AES cipher which was there main objective. They performed various analysis and simulation, for the validation of the method 
near-sink $\mathrm{CN}$ attack. The obtained results concluded the superiority of the proposed methods as compared to other methods.

Peijun Zhong, et.al, (2018)presented the extensive application of wireless sensor networks is due to the vast development and emerging technology, it has been utilized it various different fields. The sensor nodes are deployed in this network which gathered all the data, further send to base station for the communication. Various methods have been proposed so far in order to mitigate the effects of major issues faced in the network. They discussed the hot spot problem as the base station is closer to nodes of the network which tend to die earlier than other sensors. Therefore, they introduced the concept of mobile sink node, in order to remove this issue effectively [24]. The nodes of hot spot can be distributed evenly in all direction as the sink node can move along certain trajectories. In detailed, they studied the energy efficient routing method in which multiple mobile sinks was used. In the several clusters the whole network was divided in order to perform various experiments to show the effects of mobile sink on the network lifetime.

Hassan Oudani, et.al (2017)presented the study related to the wireless sensor network and faced issues in this paper in which consumption of more energy leads to decease in the network lifetime. Therefore, consumption of power by the sensor nodes in transmitting and receiving data must be minimized for which a strategy is required. There are various algorithms and methods have been proposed so far by the researchers in order to increase the network lifetime of sensor. They also developed some hierarchical protocols that lead to reduce in the network traffic toward the sink and also enhance the working capability of network. They utilized the hierarchical cluster-based approach named as LEACHES in order to perform the survey on the energyefficient [25]. In order to minimize the issue of energy consumption and maximize the lifetime of network sensor, they proposed a new method in this paper. Therefore, they performed the simulation for the evaluation of proposed method and the performance of the LEACH protocol. Matlab Simulink was used a simulator.

Nukhet Sazak, et.al (2017)presented the network in which sensor nodes are deployed randomly within the network where they can join and leave the network any time due to which significant design issues faced. There are some additional limitations faced by this network such as resource constraints, remote location, limited energy are the reasons due to which functionality of the network is degraded [26]. They proposed an active node determination method (ANDM) in this paper for WSN MAC design with the help of which, there is improvement in the energy efficiency. The integrated the ANDM with ETDMA and compared this combination with E-TDMA. The obtained results concluded the effectiveness of the proposed method which provides the usageup to $31 \%$ approx. for the optimal energy.

Harshita Jain, et.al (2017)studied an important concern of wireless sensor networks of the limited lifetime of the battery and maintaining the energy efficiency of the network. The several sensing nodes are deployed randomly in the hostile environment in order to sense the physical conditions such as temperature, pressure and so on. These sensor networks faced the issue of limited battery which is not possible for a human being to replace it as they are installed in the geographical region of hostile area. Therefore, once the battery exhausted, replacement process of battery cause major issues due to which this network is breakdown sometimes [27]. Therefore, due to these reasons, they discussed the some energy efficient routing protocols for this network. There is reduction in the packet overhead, if there is updation in the routing tables frequently, which also minimizes the energy consumption rate of the network. The used the system named as power efficient gathering in sensor information system (PEGASIS) in which combine the dynamic source routing (DSR) which helps in determining the best path, similar to the GA and BFO.

Vivek Kumar Singh, et.al (2017) presented the set of independent transducers used as the communication infrastructure and installed at different locations for the purpose of monitoring and recording the data. In the wireless sensor network, sensor nodes are deployed by which the physical conditions are sensed. The parameters sensed by these nodes are energy, temperature, humidity, pressure, direction and speed of the node [28]. There are some major challenges that has been faced by this network such as efficiency, reliability, heterogeneity, scalability, robustness, privacy and security which hampered the network working. Therefore, in order to enhance the lifetime of the sensor network, they proposed a method in this paper. This method utilized the new cluster based approach due to which reliable and energy efficient results provided by this method. The energy efficiency within the new cluster technique takes care the cluster head of the network, it is also helpful in preventing the crashes of cluster head node provide the network reliability.

Sheikh Tahir Bakhsh, et.al, (2017)proposed a new algorithm in this paper named as adaptive sleep efficient hybrid medium access control (AEH-MAC), for the wireless sensor network. This method has been widely utilized for the improvement of scheduling. The sleep times of the nodes has been adjusted by this method which also minimizes the scheduling time. The major design issue faced in scheduling the algorithm in the wireless sensor network was the allocation of the resources to different attributes. It is required to develop more mechanism, as there is wastage of more power when the listening in WSN is in the inactive state, also that method which saves the energy of the network. The major issues faced by this network are limited energy and the lesser amount of renewable resources. There is creation of delay in the network due to the utilization of fixed duty cycling that also helps in saving the energy of nodes [29]. Therefore, it is required to use the MAC protocol in the efficient manner which leads to increase ion the network lifetime further and efficiency in energy effectively. With the help of this proposed method, the sleep time is adjusted dynamically in accordance with the traffic load and wake up time of the neighbor nodes. It is required to do further improvement, for which they generate ACK packets transferred those to 
receiver as they take short packets for improving the waiting time of the source code. The each node in this proposed method took the conflict-free time slot for itself up to two-hop neighboring nodes. On the basis of proposed method, it is illustrated that proposed method has better performance in terms of runtime, number or rounds energy consumption, and slot reservation.

Fawaz Alassery, et.al (2017)presented there are various major fields in which the extensive application of wireless sensor networks has been utilized as there are large amount of sensor nodes are deployed for sensing the physical conditions of environment. The limited resources in the sensor nodes and the additional issues such as energy source, computational ability and communication bandwidth have been faced while designing the reliable smart WSN. The connectivity is guaranteed by this developed network using which lifetime of sensors batteries enhanced. They utilized the technology of virtual multiple input multiple output (V-MIMO) as a solution for the sensor nodes. This method helps in gaining the energy efficiency of smart WSNs. in this paper, they proposed a high energy efficiency smart WSN in which the advantages given by virtual MIMO technique was used [30]. The network transmission is based on the two circumstances such as first, implementation of one rechargeable relay node in the centre of the sensing field using which data packets are received from the source node. After receiving all the packets, they are decoded and forwarded to destination node. Second, in the number of clusters, the sensing field is divided contain a rechargeable relay node in each cluster. This is used to transfer the packets to the destination node in the same cluster or any other cluster. They also studied the effects of Rayleigh flat fading channels as there is enhancement in the re-transmission error of data packets. The advantages of virtual MIMOand one central/rechargeable relay node were used in order to achieve the low energy efficiency for Signal to Noise Ratio (SNR). The range of this ratio is between -20 to $20 \mathrm{~dB}$ over Rayleigh flat fading channel. The performed experiments concluded that high energy efficiency is achieved by the second scenario as compared to first as there is direct transmission in each cluster due to clustering technique and the central relay nodes.

Mehdi Kalantari, et.al (2017)reviewed numerous energy efficient routing algorithms have been proposed in this paper with time due to the emergence of new latest technologies. In this network sensor nodes are deployed by which environmental conditions are sensed and data gathered further transferred to the base station or sink node [31]. The proposed method is also useful in solving the theory of Maxwell's equations in the electrostatic similar to the set of partial differential equations. In determining the optimal paths, this method was used which gives the energy efficiency to the network. These sensors provide the geographical paths to the destination with the help of these partial differential equations. The sequence of wireless links was used to approximate the identified paths in between each pair of sensors in order to find the actual routes. Experiments were performed to validate the proposed approach and concluded that there is improvement in the network lifetime as compared to other traditional approaches.

Meirui Ren, et.al, (2017)presented this wireless sensor network has the extensive application due to which it has been utilized in almost every field in order to sense the data. Sensor nodes are deployed in the network for the collection of data which is further forwarded to the base station. The areas where they are deployed are forest, buildings, hospitals and many more. In the hostile environment, these sensor nodes are deployed for the data collection where human reach is difficult. The velocity and volume management of the large sensor nodes is necessary in order to provide the low latency time. It considered the as the difficult task as the reallocation of the sensor node is not easy. The lot of time in the network is consumed by this process as the volume of the big sensor node is minimized by the data aggregation [32]. The high density in the WSNs makes it difficult for the network to handle the large amount of the data. Therefore, they proposed a scheme in this paper using which all the issues are resolved such as latency of data aggregation and NP-hard problem. The cluster- based distribution data aggregation algorithm (DMPMC) is the scheme proposed in this paper. In both the multi- channel and multi-power WSNs, the data aggregation latency is minimized by this technique. They utilized the low transmission power for the packets transfer inside the clusters but for sending packets outside the clusterhigh power is utilized. Experiments were performed for the evaluation of proposed method which has better performance in term of lowest average latency.

Imen Ben Arbi, et.al, (2017) presented the technology using which data can be transferred from source node to the destination node using the sensor nodes which collect the data first from environment and further send it to base station, this network is termed as wireless sensor network. There is degradation in the quality of packets while transferring the data packets. Energy consumption is the major issue faced currently in this technology. For the communication purpose, this network is utilized in which the consumption of more energy is issue, due to which it is required to minimize the transfer rate of the packets from source to sink node. The development of more data reduction methods is required that helps in measuring the values of both source and sink node [33]. The difference between the sensed value and the predicted value has been measured using the threshold value. The techniques in this paper used in accordance with the type of sensed data such as for the data present in the non-linear system. In this paper, they proposed a self-exciting threshold autoregressive (SETAR) model in which nonlinear systems was used. Therefore, in order to overcome the above mentioned issues this method is used for the purpose of forecasting.

Mohamed Elshrkawey, et.al (2017)presented there are numerous applications in which this network has been utilized for the purpose of monitoring the environmental conditions. The large number of low power devices has been utilized in this wireless sensor network. Sensor nodes are deployed within the network in order to collect the information by sensing the environmental conditions after 
which transferred all the data to the base station. In this network, there are large number of clusters are present from which a single cluster head is selected. In this sensing process, initially data is sensed by sensing node, transferred to the cluster head at the end it is forwarded to base station by the cluster head. The batteries in the sensor node are inbuilt due to which they are difficult to replace. In this network, the consumption of energy is considered as the major issue as it reduces the lifetime of the network [34]. Therefore, in order to overcome all these limitations, they proposed a method in this paper. This proposed approach utilized the present energies in the cluster using which energy dissipation during network communications is minimized. Due to the selection of the cluster head in this approach it is considered as better as compared to others. They also used the TDMA technology in this method to make the process simpler. They compared the proposed method and concluded different results such as number of cluster head, decrease in energy consumption and enhancement in lifetime and on time packet delivery to base station. Therefore, simulation proved the effectiveness of the proposed method.

K. Sethu Selvam, et.al (2017) studied the major issues faced by this emerging technology extensively used in almost all applications, these issues are security and privacy. The wireless sensor network has open communication environment and limited resources due to which security issue is major concern in this network as compared to wired network. The used sensor nodes in the network are low powered devices, small in size and have low computational cost. In the field of military, hospitals, environmental monitoring and many more are some major area in which Wireless technology has been utilized. It is widely used in this area due to presence of sensor nodes that are mobile in nature. With the help of this technology, human monitoring is minimized as these are installed in the hostile environment which is not possible for them [35]. It has open environment, due to which this network is more prone to several attacks, therefore they discussed various attacks and security techniques in this paper. Also provided, different ways using which affects of these attacks are overcome. They also performed the deep analysis, on the security attacks such as active and passive. Experiments were performed and concluded that proposed mechanism has better performance in terms of low energy consumption, less memory and less computation.

Preeti Rathore, et.al (2017)presented the heterogeneous protocols of DEEC in this paper provided the effective results in terms of stability and network lifetime. They compared the performance of DEEC with other protocols in this paper and concluded the effective performance of DEEC method. In order to evaluate the performance of the proposed method, they evaluated some parameters such as, live number of node, failure number of node, stability, network lifetime and energy at different levels [36]. The sensor nodes in this network are deployed randomly which has the limited energy in each node in the initial stage. This deployed sensor nodes sense the physical conditions and gathered all data further transferred this data to the sink or Base Station (BS). In case of large numbers of rounds
$\left(R_{\max }\right)$ involved in the system leads to reduction in the energy instantly. The low battery issues breakdown all the connections due to which first node is died that provides the failed transmission of the information. They performed various experiments for the evaluation of the performance of proposed method in term of network lifetime, number of nodes alive during rounds and data packets sent to BS.

Sameer, et.al (2017)presented there are various application in our daily life in which this wireless sensor network technology has been utilized. In real time applications, for transferring the information from one location to another location they utilized the mobile nodes and sensor nodes in this network. This network also utilized one base station and one controlling station using which sink node is controlled easily therefore, it helps in transferring data from base station to destination easily. In this paper, they utilized three protocols that transferred the data easily to destination from base station. These protocols are LEACH (Low energy adaptive clustering Protocol), SEP(Stable Election Protocol) and SEP with three level of heterogeneity i.e. normal nodes, advanced nodes and intermediate nodes [37]. These methods distribute the energy to the nodes equally. They increase energy and number of advanced nodes and intermediate nodes in this paper, so that more stable protocol can be achieved easily.

Mr.R. Sathish Kumar, et.al (2017)presented the wireless sensor network an emerging technology whose extensive applications has been utilized in almost every field as it minimize the human monitoring and can be installed even in hostile environment. It faced the issue of limited resources and lonely execute simple logics in this network. The present faulty sensors within the network produced the invalid decisions and inaccurate results at the end. Therefore, for the WSNs they produced a general-purpose reasoning engine (DFLER) in this paper. The fuzzy logic technique was utilized in this method to gain the accurate and reliable results in order to fuse individual and neighborhood observations. There are large numbers of nodes present in a network that collectively formed a network after which a cluster head is selected among all which ensure the collection of all the data from all nodes after which transmitted it to base station [38]. The mainly focused on the multiple cluster head as it helps in extending the network lifetime and battery efficiency. In order to minimize the workload on the cluster head, they proposed an ECATCH algorithm in this paper that fastens data transaction as well as increase lifetime of battery. The cluster head is not responsible for balancing the energy level of node as the dynamic selection on the basis of defined parameters is done by the proposed method. The comparisons were made in this paper for the evaluation of the performance of the proposed method as compared to rest. Simulations results proved the effectiveness of the proposed method in terms of network lifetime.

Hayfa AYADI, et.al (2016)presented in this paper the study related to optimal topology such that it is possible to reduce the energy consumption rate of these networks. The critical role is played by the energy consumption in this network due to which it is necessary to minimize. There are 
various issues that degrade the functionality of network among those some critical issues were discussed in this paper such as fault detection and energy efficiency. In order to minimize these issues, researchers performed various experiments and methods developed [39].They selected the IEEE 802.15.4 standard with beacon enabled mode in this paper. The IEEE802.15.4protocol was particularly designed for the physical (PHY) layer and medium Access Control (MAC) in the WSNs. in order to present all the essential characteristic of the MAC frame in IEEE 802.15.4 they utilized the Superfame Duration (SD). This is done to minimize the energy consumption when the energy level in a battery reaches a critical level. The INETMANET/OMNeT++ simulator was utilized by them for the evaluation of the proposed method.

Soumita Sen, et.al (2016)presented the emerging technology of wireless technology in this paper in which energy consumption is the severe issue. In the sensor nodes, there is inbuilt battery which is not easy to replace as they are deployed in the hostile environment. The process of sensing, processing and transmission of data to the base station consumes large amount of energy. Therefore, researchers proposed various techniques in this paper using which the major issues of WSN are minimized [40]. They integrated the cluster and chain based data delivery approach in this paper, using which data redundancy and energy consumption issue are reduced to greater extent in static WSN. For the transmission of data at the two energy levels, the nodes are considered with dual radio. They evaluated the proposed method by doing experiments and simulations on it and demonstrated that this method increase the network lifetime andtransmission power of radio.

Mohd Zaki Shahabuddin, et.al (2016)presented there are various technologies in which IEEE standards has been utilized for the development point of view similarly in case of wireless sensor networkIEEE 802.15.4 protocol was used which has the low-rate capacities. Sometimes, even in the harsh conditions, these limited power source and communication capabilities operated for longer time. They proposed a fundamental modeling of topology control algorithm using which in the individual node of WSN the energy is conserved. This method also provides the facility of graph connectivity [41]. This proposed topology controlled the used three phases of the algorithm, first is node identification at connected at maximum transmission, the shortest algorithm with paring node is second and last is for energy conservation using which minimum power transmission per-node is calculated. Experiments were performed on the proposed method which provide the results according to which this method works locally and provide full graph connectivity. But this method is not able to minimize the WSN control overhead.

Adelcio Biazi et.al, (2016) presented the popular wireless sensor network has the major application in various fields due to the use of small sensor nodes that are mobile in nature and small power supply. The sensor nodes in the network are deployed randomly in the hostile environment such as military surveillance, hospital, forest monitoring, and many more, where human reach is difficult for monitoring every time. Sensor nodes sensed the physical conditions of the environments after which gathered all the data. This gathered data is collected by cluster head and further transferred to base station for establishing communication [42]. In the sensor node an inbuilt battery is embedded which once exhausted not replaced easily, therefore, limited battery is major concern of this network. It cost very high replacing the battery every time. In order to extend the lifetime of the network, it is necessary to use the minimum transmission power. They proposed Time Division Multiple Access (TDMA) based new technique in this paper in order to measure the accuracy of the obtained data. Therefore, there is reduction in the rate of the energy consumption due to this method. The timeslot of the sensors has been adjusted by this proposed method whenever, it is required to change in the conditions of environment. On the basis of performed experiments and obtained simulation results demonstrated the effectiveness of the proposed method as compared to the existing TDMA techniques. This method decreases the rate of energy consumption and increase the lifetime of the network.

Saleh Bouarafa et.al, (2016)presented the major issue of energy consumption in this paper by which the functionality of the network is hampered. They introduced the optimal topologyand their advantages in this paper with the help of which rate of the energy consumption in the network is minimized. Therefore, with the help of the network topology, there is reduction in the consumption of energy in the network. For the optimization of the sensor nodes, they utilized the fundamental of network topology within the network. There are various technologies that has been utilized so far such as topology directed routing, cooperating schemes, sensor coverage based topology control and many more [43]. The proposed method provides the various effective results such as increase in the lifetime of network, network monitoring, and noise free communication. They also utilized the concept of Generalized Pythagorean Theorem within this proposed method. Therefore, for the evaluation of the proposed method, they performed various experiments in this paper. The obtained simulation results proved the effectiveness of proposed method in term of network lifetime and the energy consumed by nodes. MATLAB tool was used for the simulation process in this work.

Hector Kaschel, et.al (2016)presented the widespread application of the wireless sensor network in this paper as in almost every field this wireless technology has been utilized. It provides the various advantages in the real life applications due to more developments in this network. With the help of this various advantages has been gained in this sector such as low cost, low power consumption and tiny sizes in wireless sensor nodes. Limited battery is considered as the major issue as batteries in this are inbuilt and are not easy to replace as deployed in the hostile environment [44]. Therefore, they developed and discussed various techniques in this paper that helps in achieving the energy efficiency in network. The routing protocols also plays major role in minimizing the issues faced by this network. They focused on the used routing protocols in the 
WSN in order to compare their performance with others so that their level of energy efficiency can be measured.

Mohammed Abo-Zahhad et.al, (2015) presented the various complications faced by this network such as energy consumption and energy modeling issues hampered the functionality of the wireless sensor network. In order to minimize the major issue of energy consumption, it is required to have the proper knowledge of the sources in WSNs. Therefore, for the evaluation of the communication protocols, it is required to have an accurate energy model. The parameters of the physical layer were considered in the proposed technique specially developed for energy analysis using which above mentioned issue are easily minimized. The consumed energy is transferred per payload without having error in the network using a WGN channel. It is very essential to select the transmission power appropriately, with the help of which over A WGN channel, energy-efficient communications can be achieved easily. For the optimum transmission power, this method also provided the closed-form expression. The rate of energy consumption can be minimized using the various techniques for the optimal transmission powers [45]. They evaluated the proposed methods and communication protocols in this paper for the comparison with other method, in terms of rate of energy consumed by the network. Therefore, obtained simulation results proved the effectiveness of the method in terms of increase in the lifetime of the network and reduction in the energy consumption.

\subsection{Objectives of the Study:}

Following are the various research objectives :-

1. Implement WEMER energy efficient routing protocol to increase lifetime and reduce routing overhead in wireless sensor networks

2. To improve WEMER energy efficient routing protocol to increase lifetime and reduce routing overhead in wireless sensor networks

3. Implement proposed protocol and compare with existing in terms of various parameters.

\subsection{Outline of the project}

In this report first chapter is introduction of wireless sensor networks. 1.1 overview of the topic, 1.2 specific study of the work, 1.3 literature review of research, 1.4 objectives and scope of the study. In chapter 2 theory and concepts are introduced related to research work. In this chapter problem formulation and definition of problem is described. In chapter 3 research methodology is defined, how to solve the problem. Chapter 4 includes the experiments, results of the solved problem. In chapter 5 conclusion and future work is described. At the end of the report references are included.

\section{CHAPTER 2}

Theory and Concept

\subsection{Problem Formulation}

The wireless sensor networks are the decentralized type of network in which sensor nodes sense information and pass it to base station. The energy consumption is the major issues of the sensor network due to far deployment and small size of the sensor nodes. The WEMER is the energy efficient structure free data aggregation protocol which works in the structural manner. The WEMER protocol works in the three phases, in the first phase base station send the hello message to each node in the network. The node reverts back to base station with their location and other information. In the second phase, whole network is divided into hierarchal structure based on the network density. In the third phase, the next hop node is selected based on the next node buffer size, residual energy and link strength. In this research work, WEMER protocol will be improved to reduce routing overhead in the network.

\subsubsection{Problem Definition}

Following are the various problem definitions which can be resolved in this research work:-

1. The WEMER is the protocol which work in the three phases, due to which energy hole problem can be raised. In this research work, technique will be proposed which resolve the energy hole problem

2. When the energy hole problem raised, the network throughput is reduced which can increased in this research work

\section{Chapter 3 \\ Research Methodology}

The wireless sensor networks are the self configuring type of network and size of the sensor nodes are very small. Due to which energy consumption is the major issue of wireless sensor networks. The LEACH is the energy efficient protocol which is used to reduce energy consumption of the network. The various improvements in the LEACH protocol is done in the recent times to reduce energy consumption of the network. In the proposed improvement three level of architecture is proposed in which leader nodes, cluster heads and gateway nodes are involved in the data communication The proposed technique involved following phases :-

\section{Phase 1: Cluster head selection}

The cluster head is the first phase of the network. The network is deployed with the finite number of sensor nodes. The base station is deployed at the centre of the network. The base station flood the message in the network. The base station calculates the signal strength and nodes which have signal strength above threshold value will be eligible to be selected as the cluster head. The threshold value will be defined by the below equation:-

$$
R_{C H}=R_{\min } *\left[1+\left(\frac{d_{B S}-d_{B S \min }}{d_{B S \max }-d_{B S \min }}\right)\right]
$$

In the given equation $R_{\min }$ is the radius of the cluster, $d_{B S}$ is the node distance from the base station, $d_{B S \min }$ is the minimum distance from the base station, $d_{B S \max }$ is the maximum distance from the base station.

$$
F_{C H-\text { value }}=\alpha * N_{\text {deg }}+\frac{\beta}{M S D_{\text {deg }}}+\frac{\gamma}{d_{B S}} \text { (2) }
$$

In the equaton 2 , the $N_{d e g}$ is the number of neighbor nodes of the particular node, $M S D_{\text {deg }}$ is the mean distance of all nodes in the network, $\alpha, \beta$ and $\gamma$ is the three threshold 
values whose total is 1 . The sensor node in the network generates random value which lies between 0 and 1 . When the sensor node will be selected as the cluster when it satisfy the condition given in the equation number 3

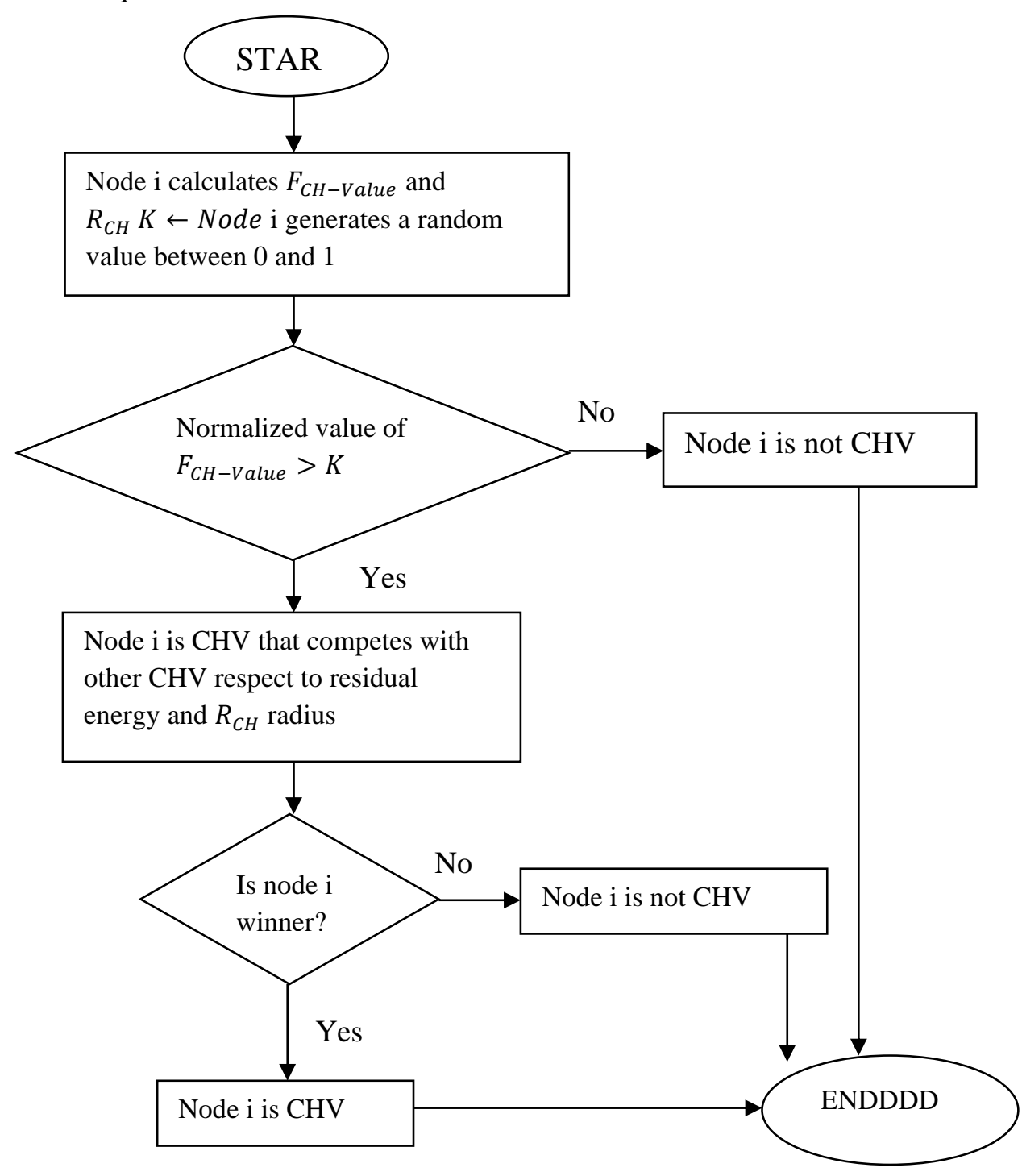

Fig 1: Flowchart of Cluster head selection Figure 3.1Cluster head selection process

Phase 2: Leader node Selection

The second phase of the proposed technique is the selection of leader nodes in the network. The nodes which are not the cluster head will be selected as the leader node. The leader nodes are responsible to collect the data from the sensor nodes and pass the sensed data to the cluster head. The volunteer leader node will be selected by the equation number 4

$F_{L N-v a l u e}=\eta * M_{\text {deg }}+\frac{\lambda}{K_{L N}}$

Mdeg is the number of leader nodes which is volunteer to selected as leader node. $K_{L N}$

Is the number of nodes which comes under the defined radius . $\eta, \lambda$ are the two constants whose total will be 1 . The nodes which are the volunteer to be selected as leader node will generate random number from 0 to 1 and nodes which satisfy condition 5 will be selected as leader node .

$$
\mathrm{K}(\mathrm{i})>F_{L N-v a l u e}
$$




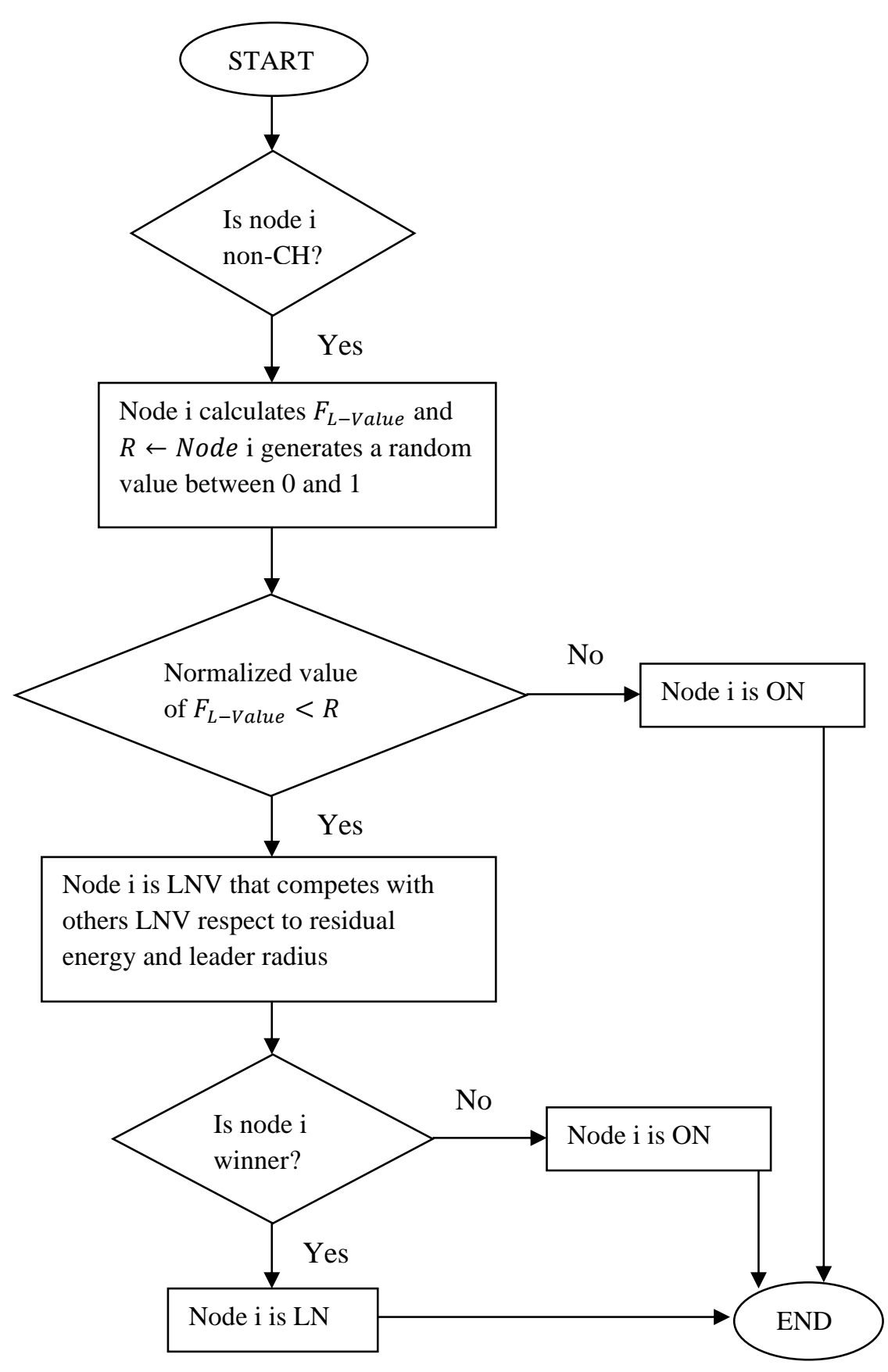

Fig 2: Leader node selection process

Figure 3.2 Leader node selection process

Phase 3: Gateway node selection

In the last phase of the algorithm, the gateway nodes are deployed in the network. The gateway nodes depends upon the total number of nodes which is described by the equation 5

Gateway ${ }_{\text {nodes }}=$ total number of nodes $/ 4$ (5)

The gateway nodes are the forth part of the total nodes. The best nodes is selected from the all gateways nodes to send data to the base station. The distance between the base station and gateway node calculated with equation 6

Distance $=\sqrt{(x(i)-x)^{2}+(y(i)-y)^{2}}$ 


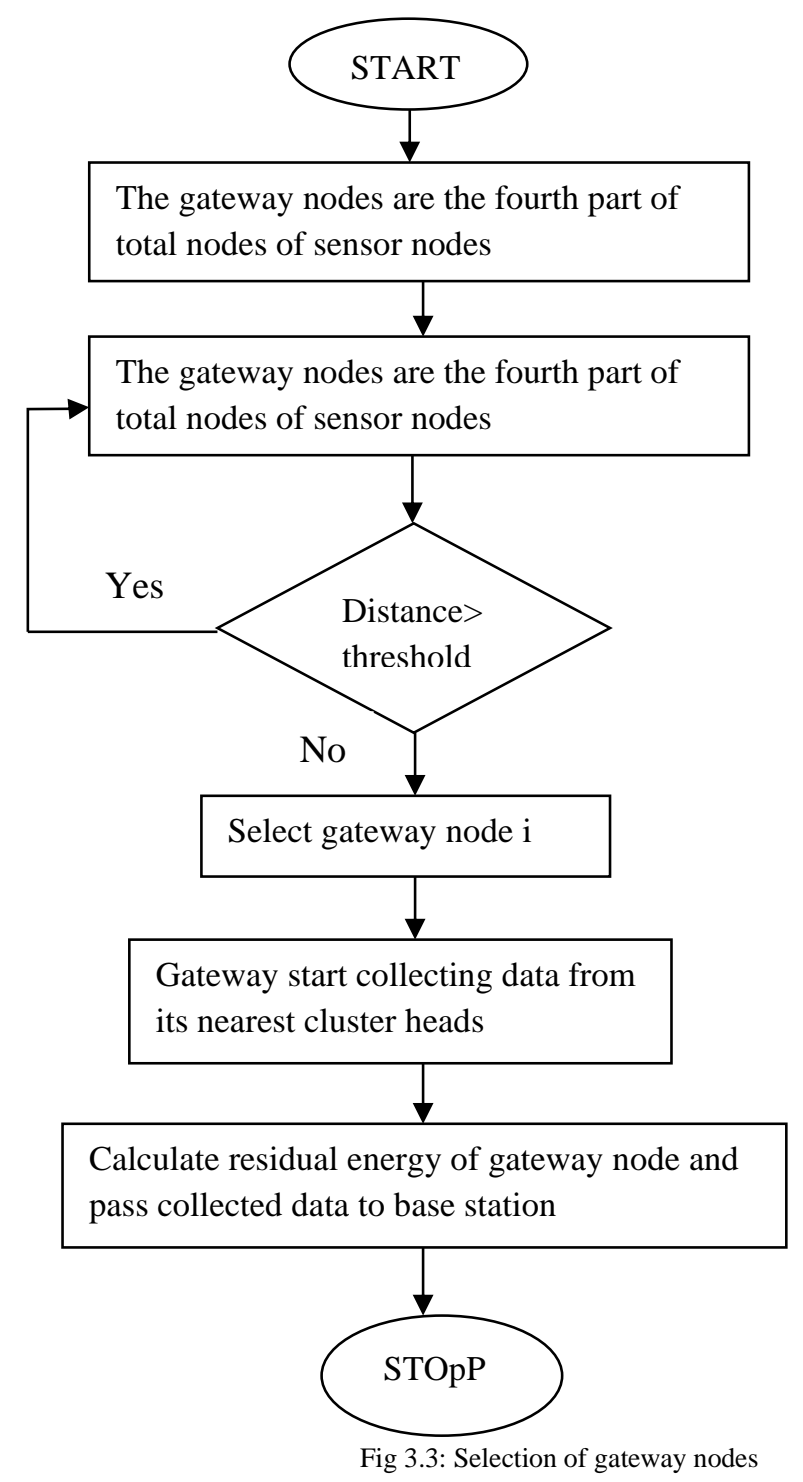

In the proposed technique, the leader nodes will aggregate data from the normal sensor nodes. The leader nodes will pass the sensed data to cluster head nodes. The cluster head ogateway node which is nearest to the base station will pass the data to the base station.

\section{CHAPTER-4}

Experiments and Results

\subsection{Tool For implementation}

The matlab is the tool which is used to perform mathematical complex computations. In this MATLAB simplified $\mathrm{C}$ is used as the programming language. The MATLAB has various inbuilt toolboxes and these toolboxes are mathematical toolbox, drag and drop based GUI, Image processing, Neural networks etc. The MATLAB is generally used to implement algorithms, plotting graphs and design user interfaces. The MATLAB has high graphics due to which it is used to simulate networks. The MATLAB has various versions by current MATLAB version is 2015. The MATLAB process elements in the form of MATRIXs and various other languages like JAVA, PYTHON and FORTAN are used in
MATLAB. The MATLAB default interface has following parts

1. Command Window:- The Command Window is the first importance part of MATLAB which is used to show output of already saved code and to execute MATLAB codes temporarily

2. WorkSpace :-The workspace is the second part of MATLAB which is used to show allocation and deallocation of MATLAB variables. The workspace is divided into three parts. The first part is MATLAB variable, variable type and third part is variable value

3. Command History :- The command history is the third part of MATLAB in which MATLAB commands are shown which are executed previously

4. Current Folder Path :- The current Folder path shows that path of the folder in which MATLAB codes are saved

Current Folder Data: - The Current Folder Data shows that data which is in the folders whose path is given in Current Folder Path 


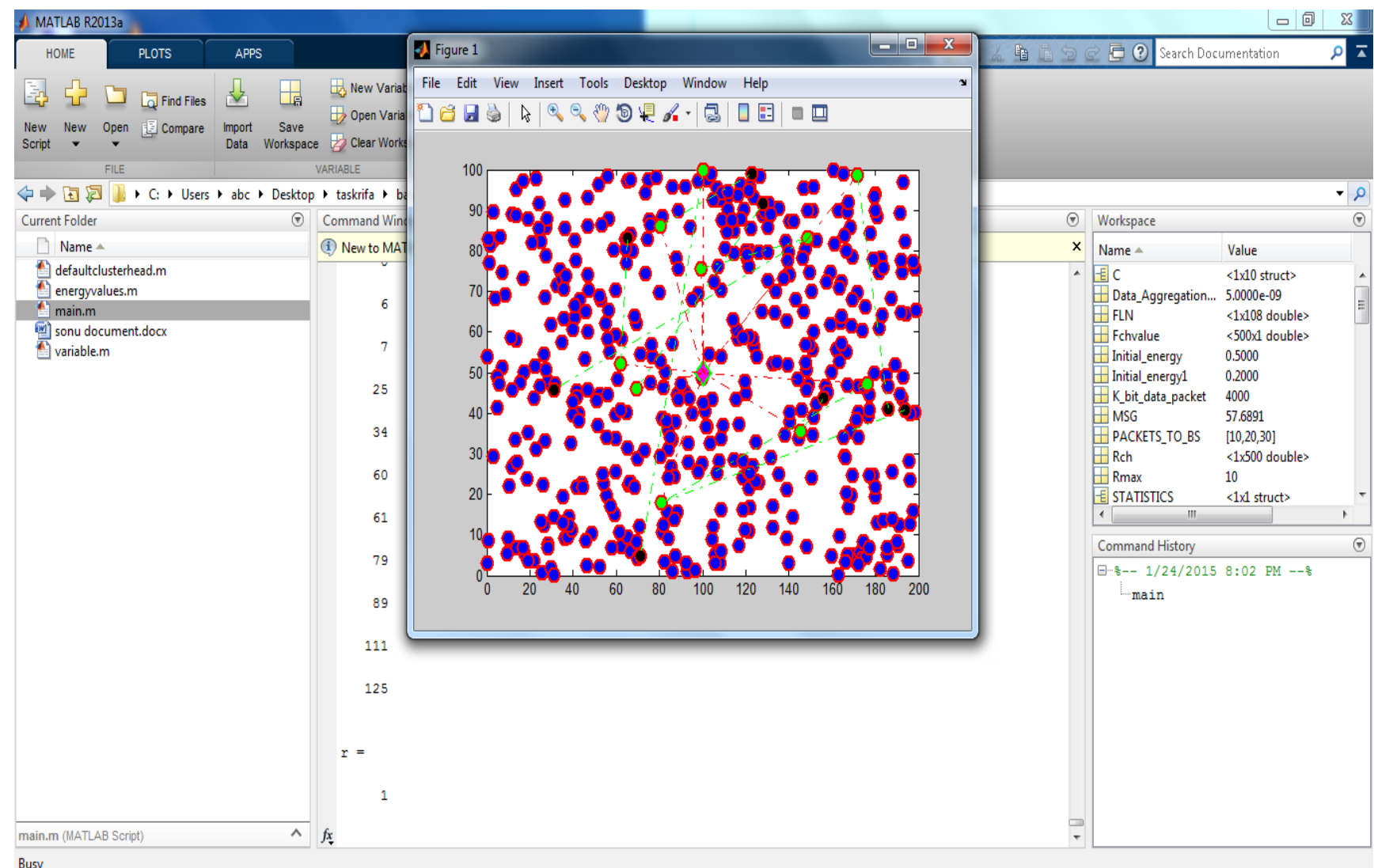

Fig 4.1: Deployment of network

As shown in figure 1, the wireless sensor network is deployed with the finite number of sensor nodes. The base station is deployed at the centre of the network.

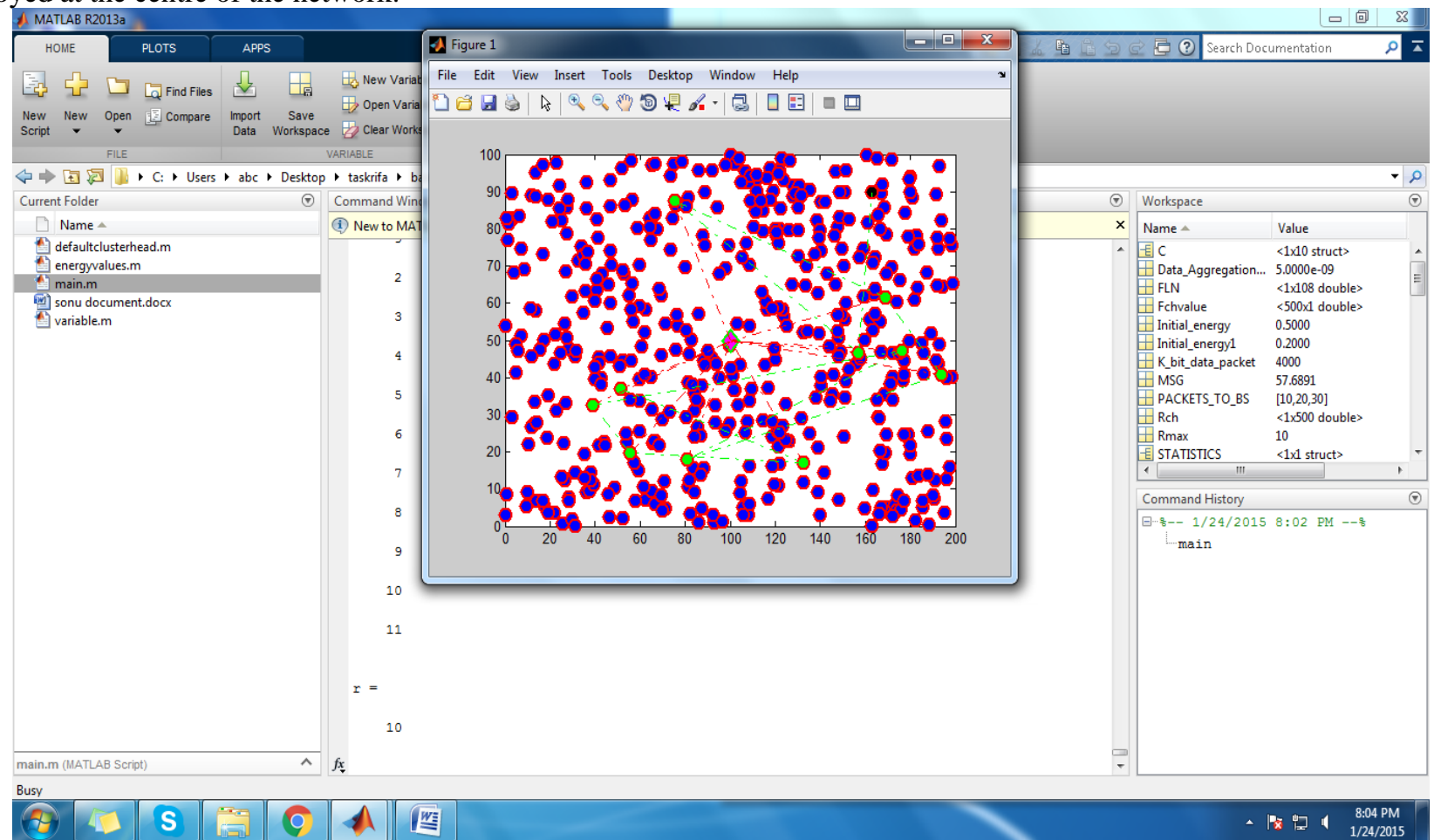

Fig 4. 2: Data Transmission

As shown in figure 2, the network is deployed with the finite number of sensor nodes. The whole network is divided into fixed size clusters and cluster head get selected in each cluster. The cluster head send data to the leader node. 


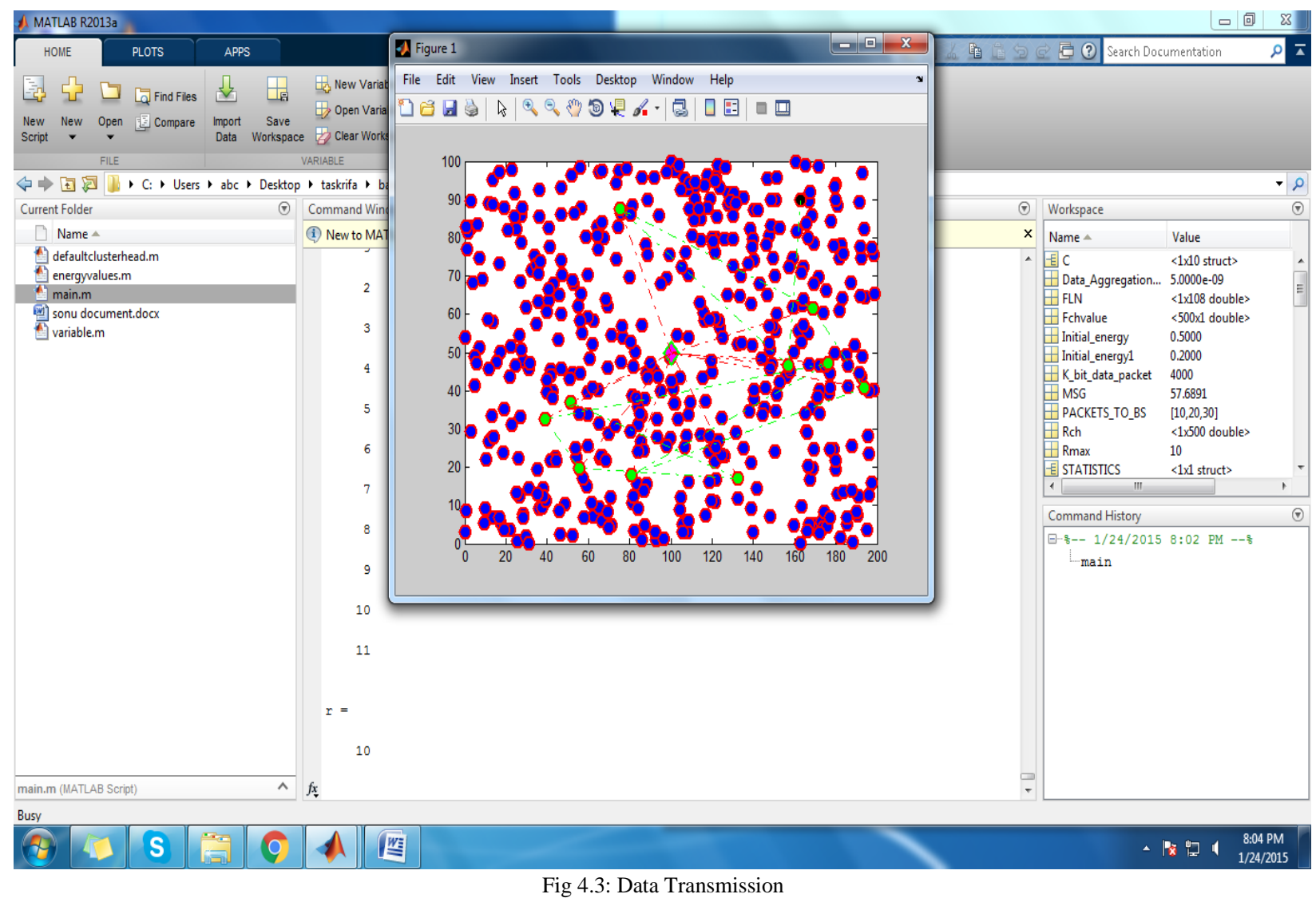

As shown in figure 3, the network is deployed with the finite number of sensor nodes. The whole network is divided into fixed size clusters and cluster head get selected in each cluster. The cluster head send data to the leader node. The leader nodes then send the data to the base station

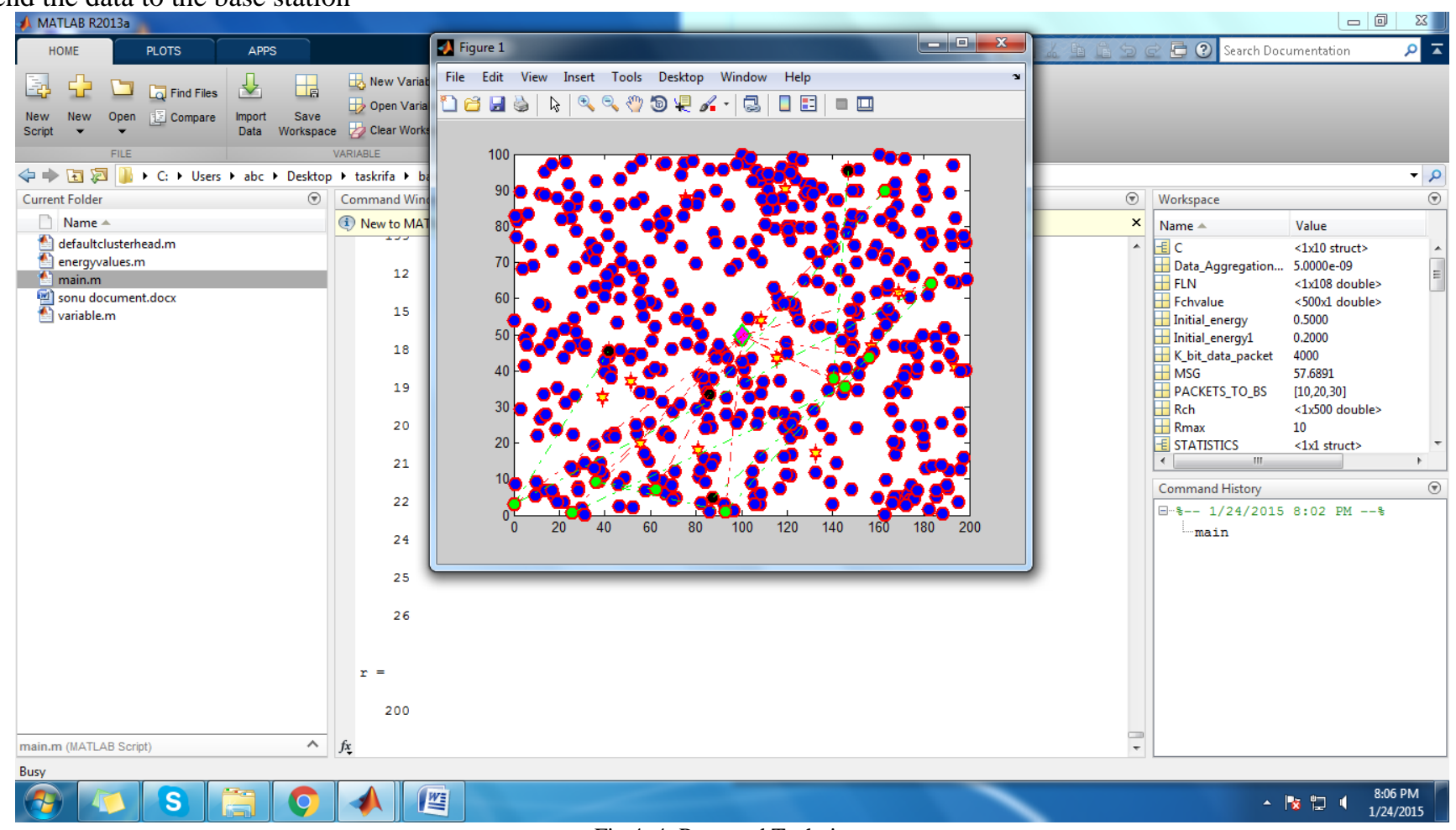

Fig 4. 4: Proposed Technique

As shown in figure 4, the whole network is deployed with the finite number of sensor nodes. The cluster heads are selected in the network. The cluster heads send data to leader nodes which send data to gateway nodes in the network 


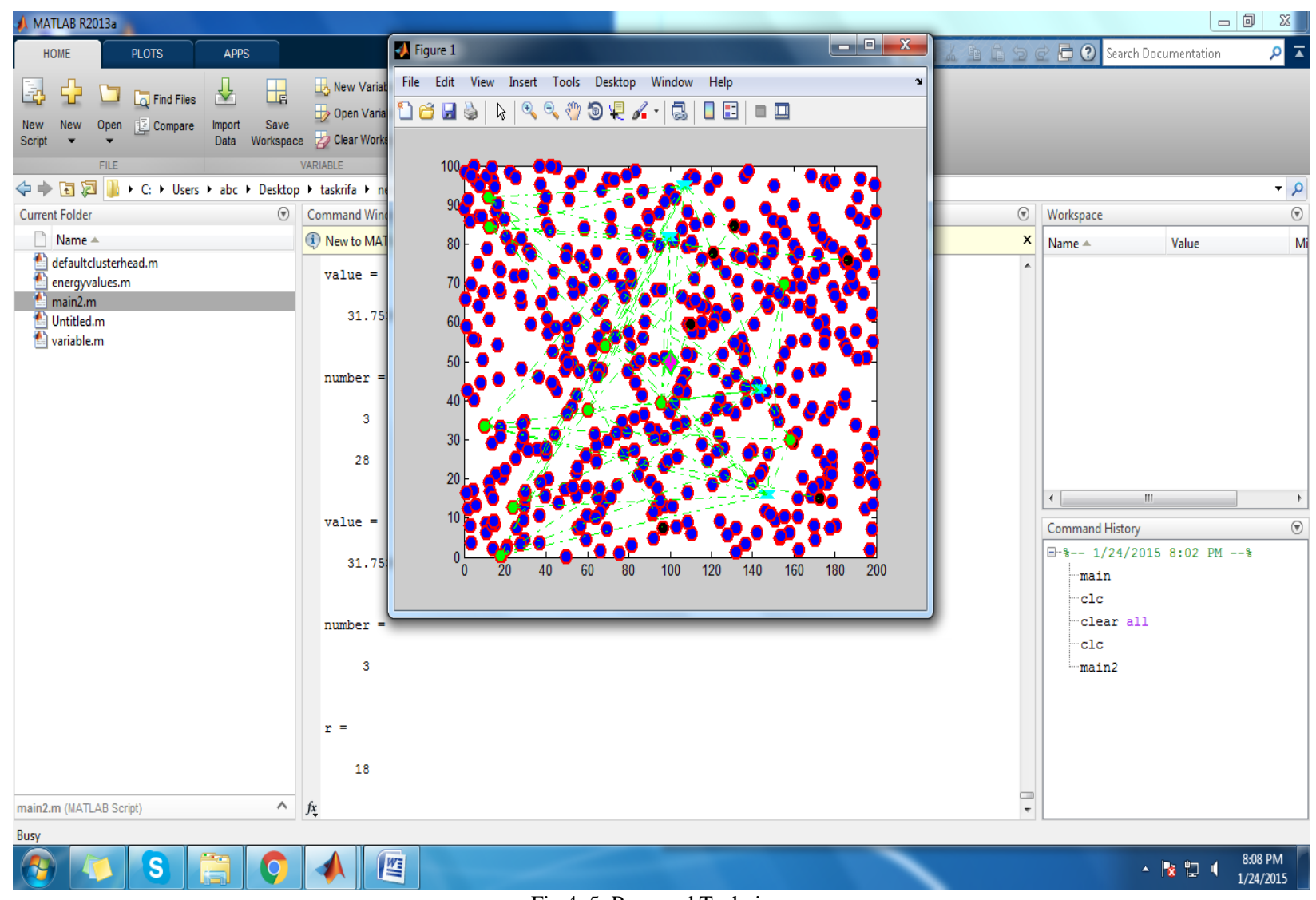

Fig 4. 5: Proposed Technique

As shown in figure 4, the whole network is deployed with the finite number of sensor nodes. The cluster heads are selected in the network. The cluster heads send data to leader nodes which send data to gateway nodes in the network

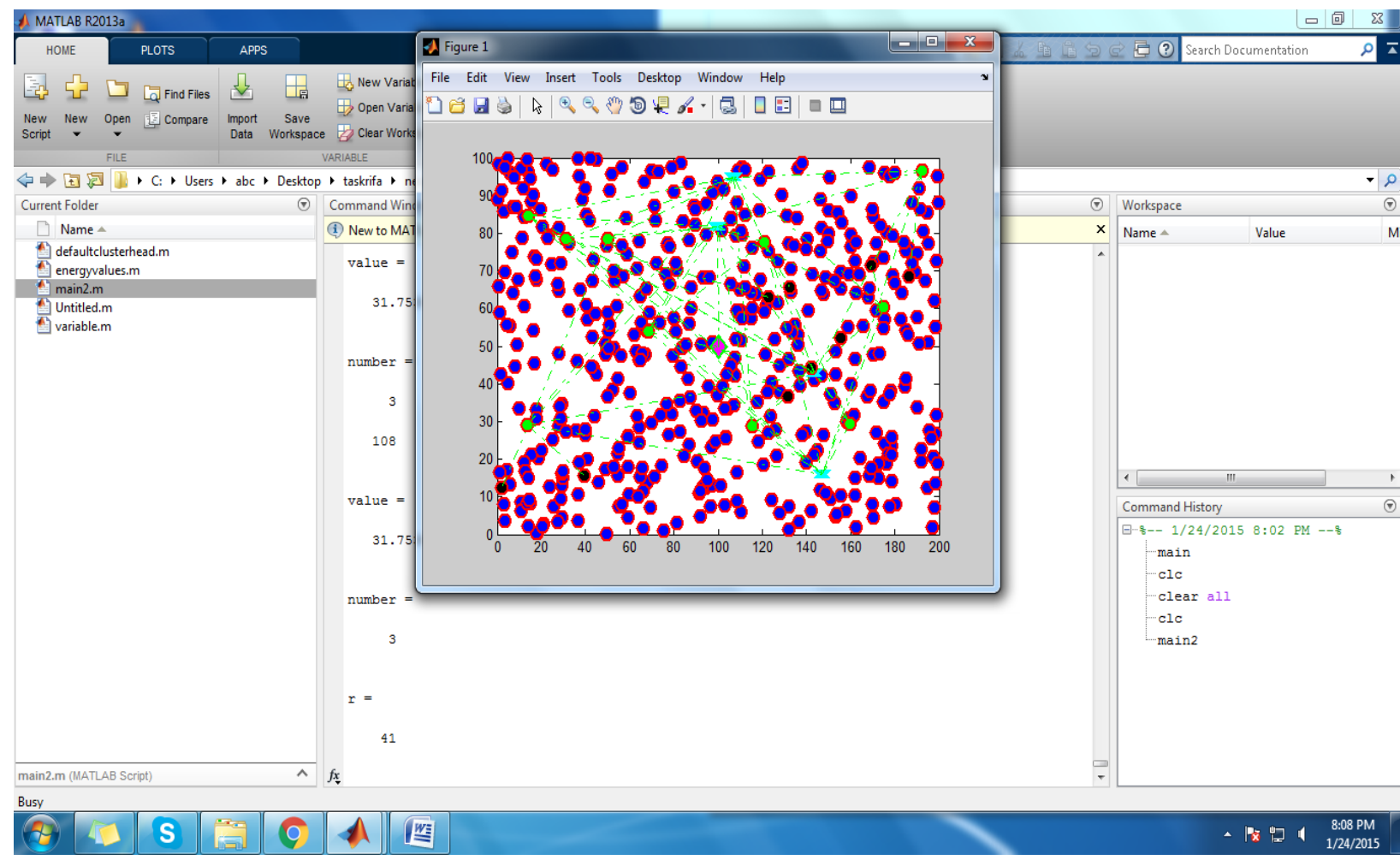

Fig 4.6: Proposed Technique

As shown in figure 4, the whole network is deployed with the finite number of sensor nodes. The cluster heads are selected in the network. The cluster heads send data to leader nodes which send data to gateway nodes in the network 


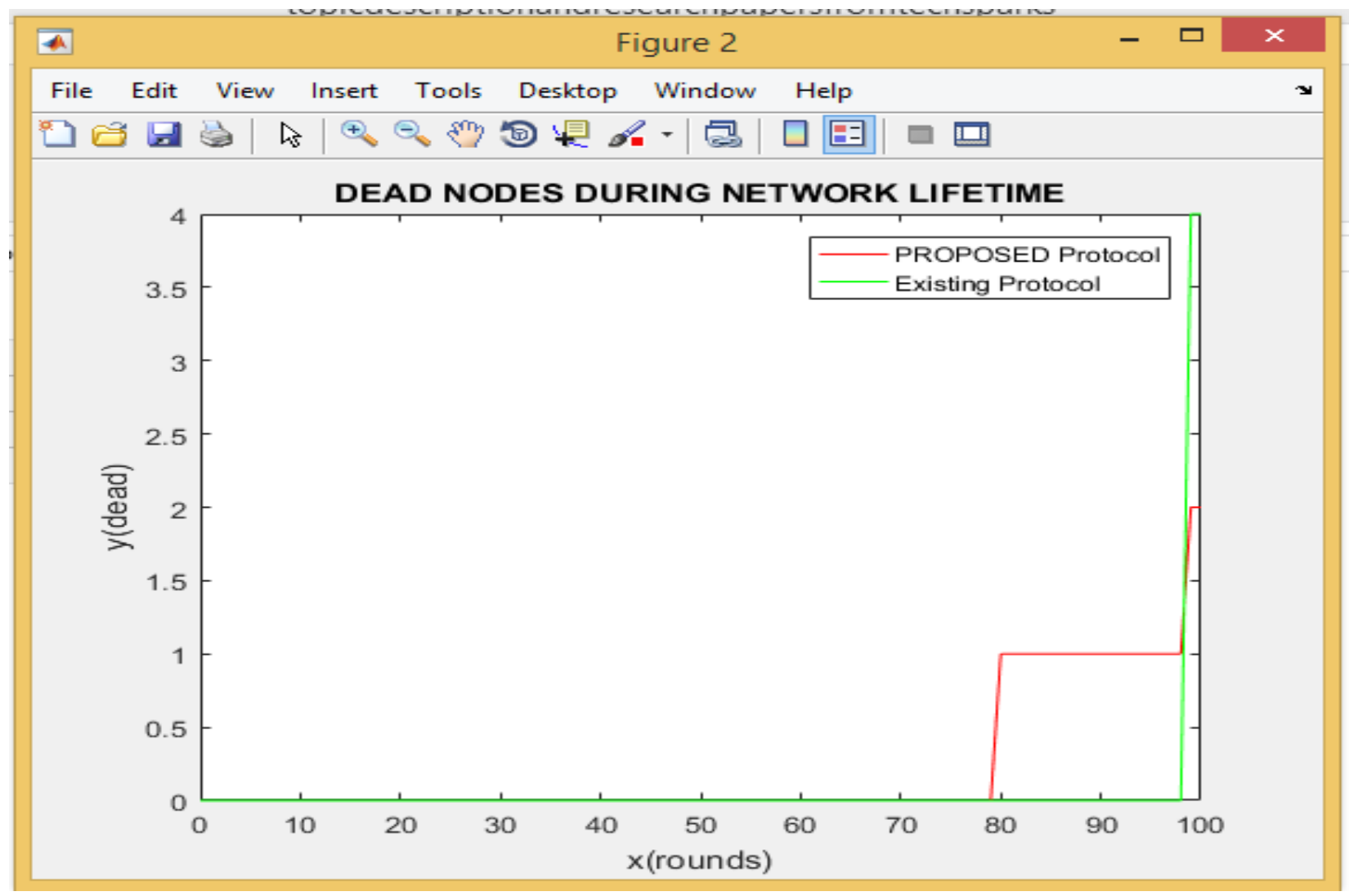

Fig 4.7: Number of dead nodes

As shown in figure 7, the number of dead nodes in the proposed and existing algorithm is compared. It is analyzed that number of dead nodes in proposed work is four and in the existing algorithm it is two.

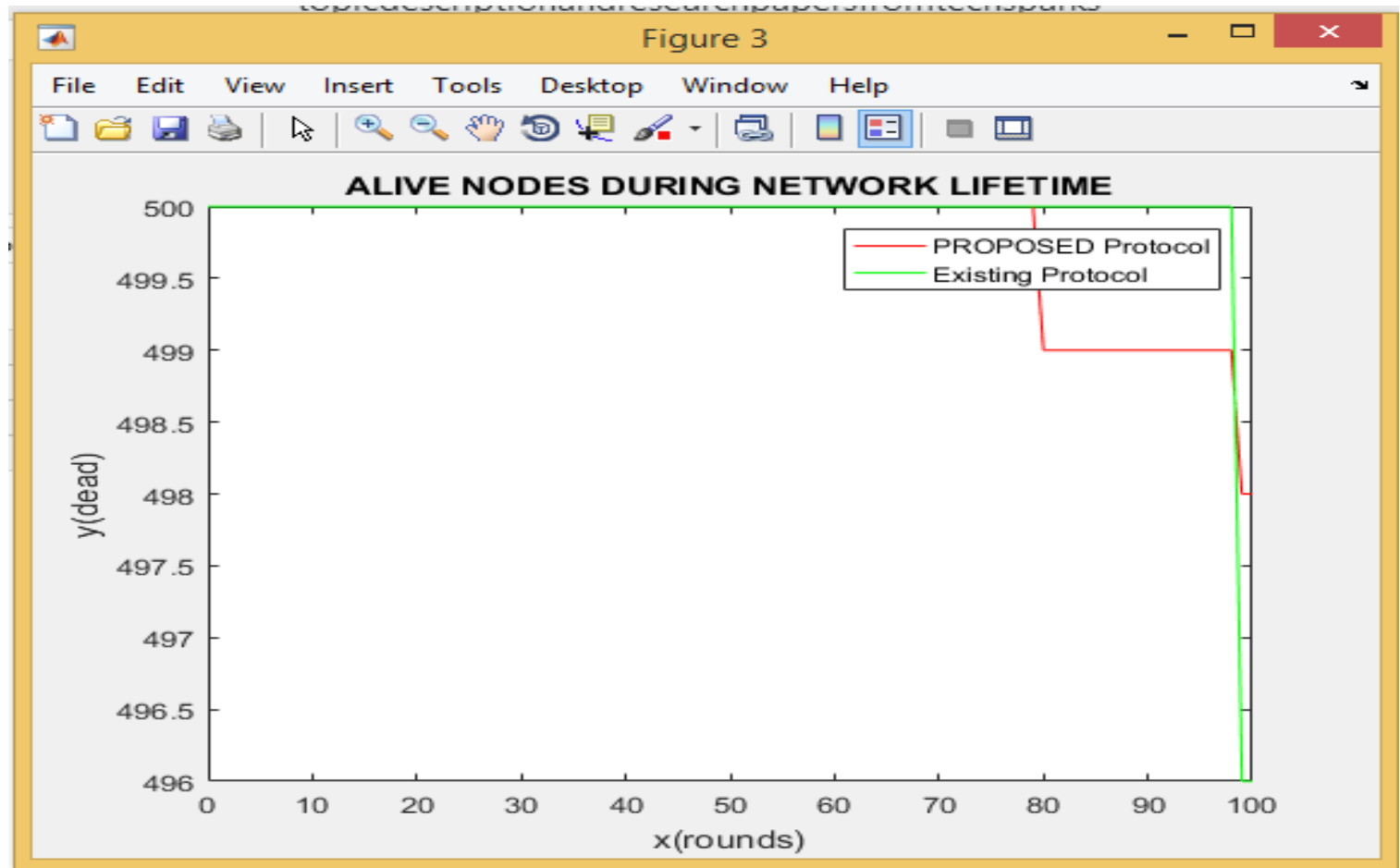

Fig 4.8: Number of alive nodes

As shown in figure 8, the number of alive nodes in the existing scheme is compared with the proposed scheme. It is analyzed that number of alive nodes are more in the proposed scheme as compared to existing scheme 


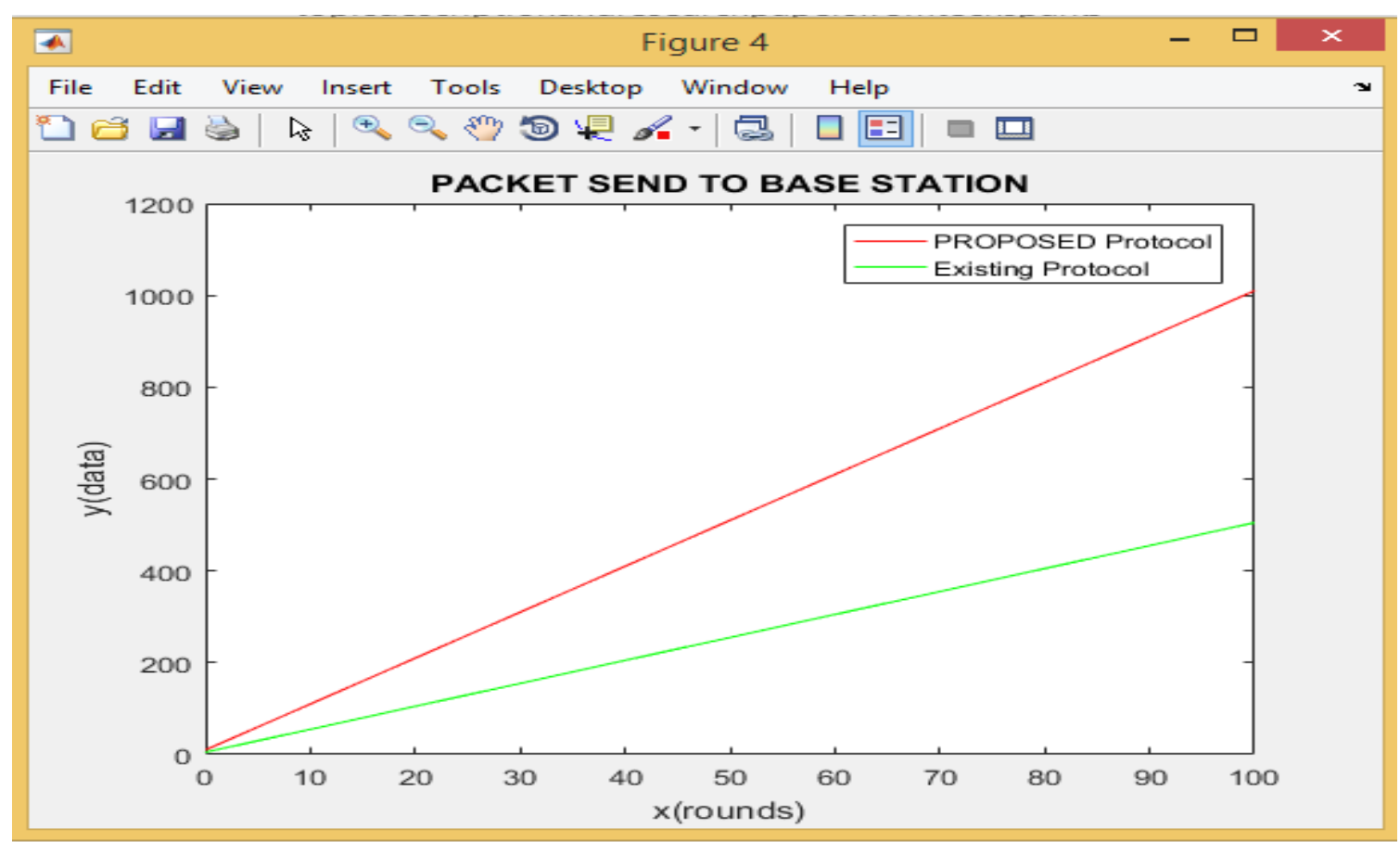

Fig 4.9: Number of packets transmitted

As shown in figure 9, the number of packet transmitted in the proposed technique is compared with the existing technique. It is analyzed that number of packets which are transmitted in the proposed technique is more as compared to existing technique

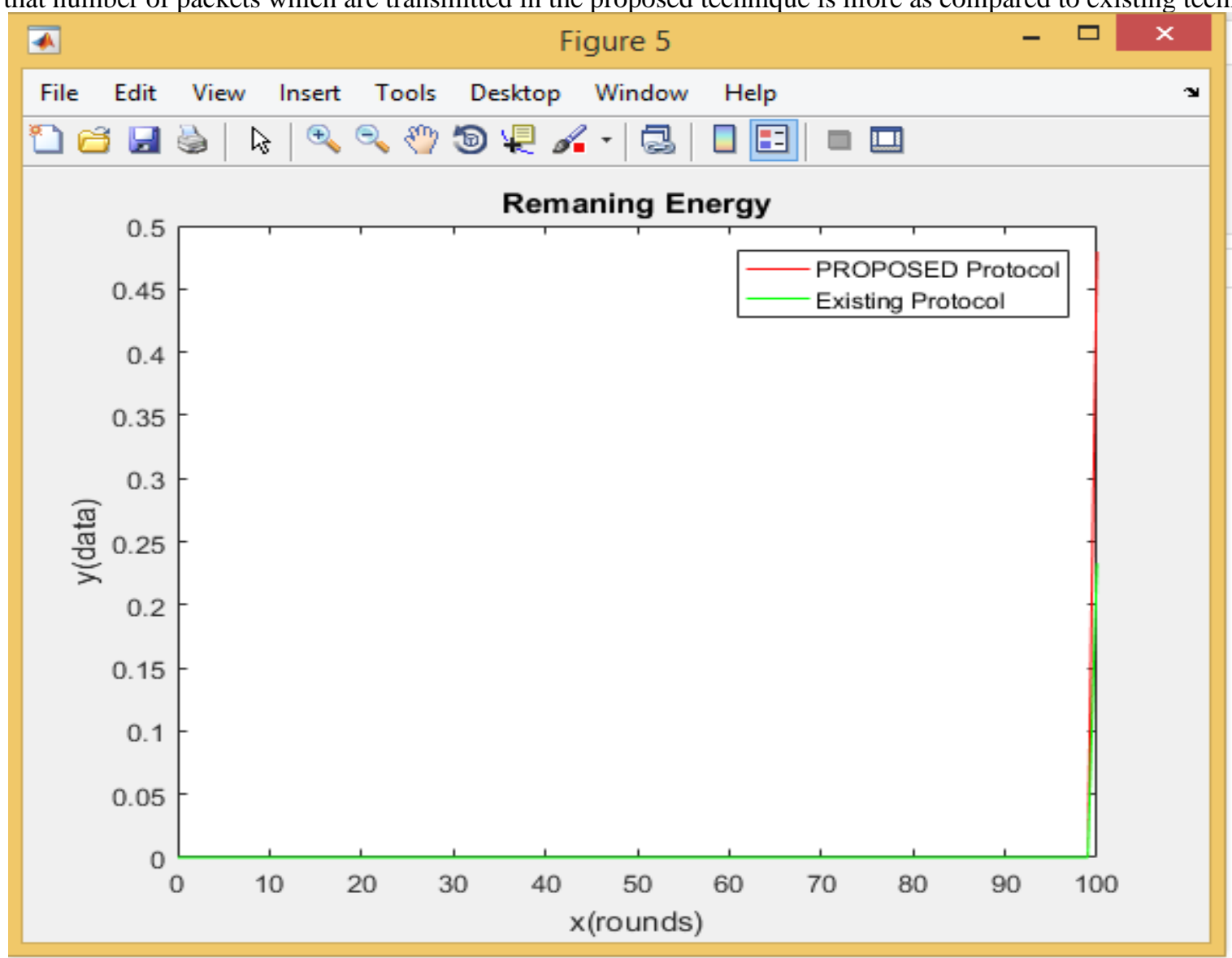

Fig 4.10: Remaining Energy

As shown in figure 10, the remaining energy of proposed technique is compared with the existing technique. It is analyzed that remaining energy in proposed technique is more as compared to existing technique 


\section{Chapter 5 \\ Conclusion and Future Work \\ 5.1 Conclusion}

The wireless sensor network is self configuring type of network which is deployed on far places to sense environmental conditions. Due to far deployment of the network energy consumption is the major issue which reduces network performance. The WEMER is the protocol in which cluster heads and leader nodes are formed in the network. The cluster head send information to leader which forward it to base station. In this research work, the WEMER protocol is improved using the gateway nodes. The cluster head send information to leader node which forward information to leader node. The leader node then forward information to gateway node. The proposed protocol is implemented in MATLAB and simulations shows upto 20 percent improvement in the results

\subsection{Future Work}

Following are the various future prospective of this research work :-

1. The proposed algorithm can be further improved compared with the other algorithms to increase lifetime of WSN

2. The proposed algorithm can be further improved to increase security of wireless sensor networks

\section{REFERENCES}

[1] Bharathidasan, A., \& Ponduru, V. A. S. (2002). Sensor networks: An overview. Department of Computer Science, University of California, Davis, CA, 95616.

[2] Somani, A. K., Kher, S., Speck, P., \& Chen, J. (2006). Distributed dynamic clustering algorithm in uneven distributed wireless sensor network. Technical Reports [DCNL-ON-2006-005], Iowa State University

[3] Mamalis, B., Gavalas, D., Konstantopoulos, C., \& Pantziou, G. (2009). Clustering in wireless sensor networks. RFID and Sensor Networks: Architectures, Protocols, Security and Integrations, Y. Zhang, LT Yang, J. Chen, eds, 324-353

[4] Bakr, B. A., \& Lilien, L. (2011, June). A quantitative comparison of energy consumption and WSN lifetime for LEACH and LEACH-SM. In Distributed Computing Systems Workshops (ICDCSW), 2011 31st International Conference on (pp. 182-191). IEEE

[5] Puccinelli, D \& Haenggi.M.(2005).Sensor Networks: applications and challenges of ubiquitous sensing.IEEE circuits and systems magazine.5(3).(pp.19-31,2005).IEEE.

[6] Dahnil, D. P., Singh, Y. P., \& Ho, C. K. (2011, February). Energy-efficient cluster formation in heterogeneous Wireless Sensor Networks: A comparative study. In Advanced Communication Technology (ICACT), 2011 13th International Conference on (pp. 746-751). IEEE.

[7] Raj, E. D. (2012). An Efficient Cluster Head Selection Algorithm for Wireless Sensor Networks-Edrleach. IOSR Journal of Computer Engineering (IOSRJCE), 2(2), 39-44.

[8] Hansen, E., Neander, J., Nolin, M., \& Björkman, M. (2006). Efficient cluster formation for sensor networks. The Online Publication Documentation System (OPUS), Mälardalen University.

[9] Xiangning, F \& Yulin, S.(2007).Improvement on LEACH Protocol of Wireless Sensor Network.International Conference on Sensor Technologies and Applications.(pp. 260 -264). IEEE

[10] Atero.F,Vinagre.J, Morgado.E \&M.R. Wilby.(2011).A Low Energy and Adaptive Architecture for Efficient Routing and Robust Mobility Management in Wireless Sensor Networks.(pp. 172 -181)IEEE.
[11] Akyildiz, I. F., Su, W., Sankarasubramaniam, Y., \& Cayirci, E (2002). Wireless sensor networks: a survey. Computer networks, 38(4), 393-422.

[12] Mendes, L. D., Rodrigues, J. J., Vasilakos, A. V., \& Zhou, L. (2011, June). Lifetime analysis of a slotted ALOHA-based wireless sensor network using a cross-layer frame rate adaptation scheme. In Communications (ICC), 2011 IEEE International Conference on (pp. 1-5). IEEE

[13] Rai, M., K., D., (2013) "Dynamic Clustering in Wireless Sensor Network using Neural Network", International Journal for Advance Research in Engineering and Technology Vol. 1, Issue II , ISSN 2320-6802.

[14] Nikodem, M., \& Wojciechowski, B. (2011, February). Upper Bounds on Network Lifetime for Clustered Wireless Sensor Networks. In New Technologies, Mobility and Security (NTMS), 2011 4th IFIP International Conference on (pp. 1-6). IEEE.

[15] Ying Miao (2005).Seminar Wireless Self-Organization Networks Application of sensor network.

[16] Pant, S., Chauhan, N., \& Kumar, P. (2010). Effective cache based policies in wireless sensor networks: A survey. Internationa Journal of Computer Applications (0975-8887) Volume, 11, 17 21

[17] Anand, D., G., Chandrakanth, H., G., and Giriprasad, M., N., D., (2012) "An Energy Efficient Distributed Protocol For Ensuring Coverage And Connectivity (E3c2) Of Wireless Sensor Networks", International Journal of Ad hoc, Sensor \& Ubiquitous Computing (IJASUC) Vol. 3, No.1

[18] Soltan, M., and Hwang, I., (2007) "Modulation-Aware Energy Balancing in Hierarchical Wireless Sensor Networks", EURASIP Journal on Wireless Communications and Networking, pp. 355 359.

[19] Bharathidasan, A., \& Ponduru, V. A. S. (2002). Sensor networks: An overview. Department of Computer Science, University of California, Davis, CA, 95616

[20] Akyildiz, I. F., Su, W., Sankarasubramaniam, Y., \& Cayirci, E. (2002). Wireless sensor networks: a survey. Computer networks, 38(4), 393-422.

[21] Oyman, E. I., \& Ersoy, C. (2004, June). Multiple sink network design problem in large scale wireless sensor networks. In Communications, 2004 IEEE International Conference on (Vol. 6 , pp. 3663-3667). IEEE

[22] Sohrabi, K., Gao, J., Ailawadhi, V., \& Pottie, G. J. (2000). Protocols for self-organization of a wireless sensor network. IEEE personal communications, 7(5), 16-27

[23] Cheng, Y., Li, H., Wan, P. J., \& Wang, X. (2012). Wireless mesh network capacity achievable over the csma/ca mac. Vehicular Technology, IEEE Transactions on, 61(7), 3151-3165.

[24] Salzmann, J., Behnke, R., \& Timmermann, D. (2011, March). Hex-MASCLE-hexagon based clustering with self healing abilities. In Wireless Communications and Networking Conference (WCNC), 2011 IEEE (pp. 528-533). IEEE.

[25] Xu, J., Jin, N., Lou, X., Peng, T., Zhou, Q., \& Chen, Y. (2012, May). Improvement of LEACH protocol for WSN. In Fuzzy Systems and Knowledge Discovery (FSKD), 2012 9th International Conference on (pp. 2174-2177). IEEE.

[26] Younis, O., Krunz, M., \& Ramasubramanian, S. (2006). Node clustering in wireless sensor networks: recent developments and deployment challenges. Network, IEEE, 20(3), 20-25.

[27] Sharma, S., \& Kaur, B. (2010). Literature and Solution to packet collision in network in wireless communication. Networks

[28] Issariyakul, T., \& Hossain, E. (2009). Transport Control Protocols Part 2-Transmission Control Protocol (TCP). In Introduction to Network Simulator NS2 (pp. 1-43). Springer US

[29] Gouvy, N., Hamouda, E., Mitton, N., \& Zorbas, D. (2013, April). Energy efficient multi-flow routing in mobile Sensor Networks. In Wireless Communications and Networking Conference (WCNC), 2013 IEEE (pp. 1968-1973). IEEE

[30] Yang, M., Qin, Q. "A routing protocol algorithm in wireless sensor networks", Comput. Eng. Appl., 2004, 32, pp. 130-131

[31] Huang, S., Cao, Y., Wang, Y. "Routing technology in wireless sensor networks", Comput. Eng. Appl., 2004, 19, pp. 123-126

[32] Smaragdakis, G., Matta, I., Bestavros, A.: 'SEP: A stable election protocol for clustered heterogeneous wireless sensor networks'. Proc. of 2nd Int. Workshop on Sensor and Actor Network Protocol and Applications, SANPA, 2004 
[33] Abdelbari Ben Yagouta, Maher Jabberi, Bechir Ben Gouissem, "Impact of Sink Mobility on Quality of Service Performance and Energy Consumption in Wireless Sensor Network with Cluster Based Routing Protocols", 2017 IEEE/ACS 14th International Conference on Computer Systems and Applications

[34] Jie Huang, "Research on Balanced Energy Consumption of Wireless Sensor Network Nodes Based on Clustering Algorithm", 2017 International Conference on Computer Network, Electronic and Automation

[35] Yu Shaojun, Lin Li, Li Xiaozhen, "Dynamic energy consumption analysis and test of node in wireless sensor networks", 2017 IEEE 13th International Conference on Electronic Measurement \& Instruments

[36] Tenager Mekonnen, Mika Komu, Roberto Morabito, Tero Kauppinen, Erkki Harjula, Timo Koskela, and Mika Yliantila, "Energy Consumption Analysis of Edge Orchestrated Virtualized Wireless Multimedia Sensor Networks", 2017, IEEE, volume 6, pp- 5090-5100

[37] H.Oudani, S.Krit, M. Kabrane , K. Karimi, M. Elasikri, K. Bendaoud, H. El Bousty, L. Elmaimouni, "Minimize Energy Consumption in Wireless Sensor Network Using Hierarchical Protocols", ICEMIS, 2017

[38] M. Kabrane, S. Krit, L. Elmaimouni, K. Bendaoud, H. Oudani,, M. Elasikri, K. Karimi and H. El Bousty, "Smart Cities: Energy Consumption in Wireless Sensor Networks for Road Traffic Modeling Using Simulator SUMO”, 2017, IEEE

[39] M. Benaddy, B. El Habil, M. El Ouali, O. El Meslouhi, S. Krit, “A mutlipath routing algorithm for wireless sensor networks under distance and energy consumption constraints for reliable data transmission", 2017, IEEE

[40] K. Praghash, R. Ravi, "Energy Consumption Architecture for Wireless Sensor Networks With Different Clusters", 2017 Third International Conference on Science Technology Engineering \& Management (ICONSTEM)

[41] Pallavi Yarde, Sumit Srivastava, Kumkum Garg, "A modified energy efficient protocol for optimization of dead nodes and energy consumption in wireless sensor networks", 2017 Eleventh International Conference on Sensing Technology (ICST)

[42] Abdelbari Ben Yagouta, Rahma Gantassi, Bechir Ben Gouissem, "Compromises between Energy Consumption and Quality of Service Metrics in Wireless Sensor Networks with Mobile Sink and Cluster based Routing Protocols", 2017, IEEE

[43] Qasem Abu Al-Haija, Hesham Enshasy, Abdullah Smadi, "Estimating Energy Consumption of Diffie Hellman Encrypted Key Exchange (DH-EKE) for Wireless Sensor Network", 2017 IEEE INTERNATIONAL CONFERENCE ON INTELLIGENT TECHNIQUES IN CONTROL, OPTIMIZATION AND SIGNAL PROCESSING

[44] Yang Liu, and Jing Li, "Linear Precoding to Optimize Throughput, Power Consumption and Energy Efficiency in MIMO Wireless Sensor Networks", 2018, IEEE

[45] Yi-Hua Zhu, Ertao Li, and Kaikai Chi, "Encoding Scheme to Reduce Energy Consumption of Delivering Data in Radio Frequency Powered Battery-free Wireless Sensor Networks", 2015, IEEE TRANSACTIONS ON VEHICULAR TECHNOLOGY

[46] Mohammed Abo-Zahhad, Mohammed Farrag and Abdelhay Ali, "Modeling and Minimization of Energy Consumption in Wireless Sensor Networks", 2015, IEEE

[47] Nabih Alaouil, Jean-Pierre Cances, Vahid Meghdadi, "Energy Consumption In Wireless Sensor Networks for network coding structure and ARQ protocol", 1 st International Conference on Electrical and Information Technologies ICEIT'2015

[48] Dr. Zainab Tawfeeq Alisa, Hussein A. Nassrullah, "Minimizing Energy Consumption in Wireless Sensor Networks using Modified Genetic Algorithm and an Energy Balance Filter", 2016 Al-Sadeq International Conference on Multidisciplinary in IT and Communication Science and Applications (AIC-MITCSA)

[49] Fernando R. Almeida Jr., Angelo Brayner, Joel J.P.C. Rodrigues $\dagger$, Jose E. Bessa Maia, "Fractal Clustering and Similarity Measure: Two New Approaches for Reducing Energy Consumption in Wireless Sensor Networks", 2016, IEEE

[50] Yao Lu , Pierre Kuonen, Beat Hirsbrunner, Mingwei Lin, "Benefits of data aggregation on energy consumption in wireless sensor networks", 2017, IET Communications Vol. 11 Iss. 8, pp. 1216-1223

[51] Omar Abdulwahabe Mohamad, "Improved Performance of Energy Consumption in Wireless Sensor Networks Based on ZigBee Transceiver Protocol", ECAI 2016 - International Conference - 8th Edition

[52] Viktor Toldov, Roman Igual-Perez, Rahul Vyas, Alexandre Bo'e, Laurent Clavier, Nathalie Mitton, "Experimental Evaluation of Interference Impact on the Energy Consumption in Wireless Sensor Networks", 2016, IEEE

[53] Wenliang Wu, Naixue Xiong, Chunxue Wu, "Improved clustering algorithm based on energy consumption in wireless sensor networks", 2017, IET Netw., The Institution of Engineering and Technology

[54] Neha V. Deshmukh, Prof. A. V. Deorankar, "Minimizing Energy Consumption in Transmission Efficient Wireless Sensor Network", 2016, International Conference on Advances in Electrical, Electronics, Information, Communication and Bio-Informatics (AEEICB16)

[55] Veervrat Singh, Rajiv K. Tripathi, Y. N. Singh and Nishchal K. Verma, "Tradeoff between energy consumption and lifetime in two tiered wireless sensor networks", 2015, IEEE

[56] Ashfaq Ahmad, Nadeem Javaid, Muhammad Imran, Mohsen Guizani, Ahmad A. Alhamed, "An advanced energy consumption model for terrestrial wireless sensor networks", 2016, IEEE

[57] Tuan-Duc Nguyen, "Energy efficient wireless sensor network and low power consumption station design for an urban water leve monitoring system", 2016 3rd National Foundation for Science and Technology Development Conference on Information and Computer Science 